TRANSACTIONS OF THE

AMERICAN MATHEMATICAL SOCIETY

Volume 350, Number 11, November 1998, Pages 4473-4498

S 0002-9947(98)02091-1

\title{
TRIGONOMETRIC MOMENT PROBLEMS FOR ARBITRARY FINITE SUBSETS OF $\mathrm{Z}^{n}$
}

\author{
JEAN-PIERRE GABARDO
}

\begin{abstract}
We consider finite subsets $\Lambda \subset \mathbf{Z}^{n}$ satisfying the extension property, i.e. the property that every collection $\left\{c_{\mathbf{k}}\right\}_{\mathbf{k} \in \Lambda-\Lambda}$ of complex numbers which is positive-definite on $\Lambda$ is the restriction to $\Lambda-\Lambda$ of the Fourier coefficients of some positive measure on $\mathbf{T}^{n}$. A simple algebraic condition on the set of trigonometric polynomials with non-zero coefficients restricted to $\Lambda$ is shown to imply the failure of the extension property for $\Lambda$. This condition is used to characterize the one-dimensional sets satisfying the extension property and to provide many examples of sets failing to satisfy it in higher dimensions. Another condition, in terms of unitary matrices, is investigated and is shown to be equivalent to the extension property. New two-dimensional examples of sets satisfying the extension property are given as well as explicit examples of collections for which the extension property fails.
\end{abstract}

\section{Notation}

If $\Lambda$ is a finite set contained in $\mathbf{Z}^{n}$, we will denote by $|\Lambda|$ its cardinality and by $\Lambda$ $\Lambda$ the difference set defined by $\Lambda-\Lambda=\left\{\lambda-\lambda^{\prime}, \lambda, \lambda^{\prime} \in \Lambda\right\}$. $\mathbf{T}^{n}$ will be identified with the set of $n$-tuples of complex numbers of modulus 1, i.e. $\mathbf{T}^{n}=\left\{\left(z_{1}, \ldots, z_{n}\right),\left|z_{i}\right|=\right.$ $1, i=1, \ldots, n\}$. If $\mathbf{z}=\left(z_{1}, \ldots, z_{n}\right) \in \mathbf{T}^{n}$ and $\mathbf{k}=\left(k_{1}, \ldots, k_{n}\right) \in \mathbf{Z}^{n}$, we will use the notation $\mathbf{z}^{\mathbf{k}}$ for the complex number $z_{1}^{k_{1}} \ldots z_{n}^{k_{n}}$. For a fixed finite set $\Lambda \subset \mathbf{Z}^{n}$, we will denote by $\Pi_{\Lambda}$ the set of all trigonometric polynomials with non-zero Fourier coefficients restricted to $\Lambda$, i.e. $\Pi_{\Lambda}=\left\{P(\mathbf{z})=\sum_{\mathbf{k} \in \Lambda} a_{\mathbf{k}} \mathbf{z}^{\mathbf{k}}, a_{\mathbf{k}} \in \mathbf{C}, \mathbf{k} \in \Lambda\right\}$, and we will always consider the elements of $\Pi_{\Lambda}$ as functions defined on $\mathbf{T}^{n} \cdot \mathbf{M}\left(\mathbf{T}^{n}\right)$ will be the set of Borel measures on $\mathbf{T}^{n}$. Finally, $\mathbf{M}_{k}$ denotes the set of $k \times k$ matrices with complex entries.

\section{INTRODUCTION}

The problem of the extension of positive-definite functions has a long history, starting with the fundamental paper by M. G. Krein $([\mathrm{Kr}])$ on the possibility of extending a continuous positive-definite function defined on a finite interval to the whole real line. Different facets of this problem have, since then, been investigated by many authors in various settings (e.g. [Ak], [AK], [CF], [De], [Ga1], [Ga2], [Jo1], [Jo2], [La1], [La2], [Lan], [Sas1]-[Sas3]), and, yet, many fundamental problems are

Received by the editors June 15, 1996.

1991 Mathematics Subject Classification. Primary 42A70, 44A60.

Key words and phrases. Tight frames, evaluation polynomials, representing measures, positivedefinite, extension problem.

The author was supported by NSERC grant OGP0036564.

(C)1998 American Mathematical Society 
still largely open in this area. The present paper deals with a discrete, multidimensional version of Krein's problem. Let us consider a finite set $\Lambda \subset \mathbf{Z}^{n}$. We say that a collection $\left\{c_{\mathbf{k}}\right\}_{\mathbf{k} \in \Lambda-\Lambda}$ of complex numbers, indexed by the difference set $\Lambda-\Lambda$, is positive-definite (p.d.) on $\Lambda$ if, for any collection $\left\{\xi_{\mathbf{k}}\right\}_{\mathbf{k} \in \Lambda}$ of complex numbers, indexed by $\Lambda$, we have

$$
\sum_{\mathbf{k}, \mathbf{l} \in \Lambda} c_{\mathbf{l}-\mathbf{k}} \xi_{\mathbf{k}} \overline{\xi_{\mathbf{l}}} \geq 0
$$

The collection $\left\{c_{\mathbf{k}}\right\}_{\mathbf{k} \in \Lambda-\Lambda}$ will be called strictly positive-definite (s.p.d.) on $\Lambda$ if equality in (2.1) only holds when all the $\xi_{\mathbf{k}}$ 's are zero. If $\mu \in \mathbf{M}\left(\mathbf{T}^{n}\right)$ is a positive measure and $c_{\mathbf{k}}$ is defined to be the $\mathbf{k}$-th Fourier coefficient of $\mu$, i.e.

$$
c_{\mathbf{k}}=\frac{1}{(2 \pi)^{n}} \int_{\mathbf{T}^{n}} \mathbf{z}^{-\mathbf{k}} d \mu, \quad \mathbf{k} \in \Lambda-\Lambda,
$$

then, letting $P(\mathbf{z})=\sum_{\mathbf{k} \in \Lambda} \xi_{\mathbf{k}} \mathbf{z}^{\mathbf{k}} \in \Pi_{\Lambda}$, we have that

$$
\begin{aligned}
\frac{1}{(2 \pi)^{n}} \int_{\mathbf{T}^{n}}|P(\mathbf{z})|^{2} d \mu & =\sum_{\mathbf{k}, \mathbf{l} \in \Lambda}\left\{\frac{1}{(2 \pi)^{n}} \int_{\mathbf{T}^{n}} \mathbf{z}^{\mathbf{k}-\mathbf{l}} d \mu\right\} \xi_{\mathbf{k}} \overline{\xi_{\mathbf{l}}} \\
& =\sum_{\mathbf{k}, \mathbf{l} \in \Lambda} c_{\mathbf{l}-\mathbf{k}} \xi_{\mathbf{k}} \overline{\xi_{\mathbf{l}}} \geq 0,
\end{aligned}
$$

and, thus, the restriction to $\Lambda-\Lambda$ of the sequence (indexed by $\mathbf{Z}^{n}$ ) of the Fourier coefficients of a positive measure provides us with an example of a collection which is positive-definite on $\Lambda$. If, for a collection $\left\{c_{\mathbf{k}}\right\}_{\mathbf{k} \in \Lambda-\Lambda}$, there is a positive measure $\mu$ on $\mathbf{T}^{n}$ satisfying (2.2), then $\mu$ will be called a representing measure associated with the collection $\left\{c_{\mathbf{k}}\right\}_{\mathbf{k} \in \Lambda-\Lambda}$ and the collection $\left\{c_{\mathbf{k}}\right\}_{\mathbf{k} \in \Lambda-\Lambda}$ will be called extendable. Note that if a collection $\left\{c_{\mathbf{k}}\right\}_{\mathbf{k} \in \mathbf{Z}^{n}}$ indexed by $\mathbf{Z}^{n}$ satisfies (2.1) for all finite sets $\Lambda \subset \mathbf{Z}^{n}$, then this collection is actually the sequence of Fourier coefficients of some positive measure on $\mathbf{T}^{n}$ by Bochner's theorem. The fundamental question that we will be addressing in this paper is whether or not a set $\Lambda \subset \mathbf{Z}^{n}$ has the property that any collection positive-definite on $\Lambda$ is the restriction to $\Lambda-\Lambda$ of the Fourier coefficients of some positive Borel measure on $\mathbf{T}^{n}$.

Definition 2.1. A finite set $\Lambda \in \mathbf{Z}^{n}$ is said to have the extension property if, for any collection $\left\{c_{\mathbf{k}}\right\}_{\mathbf{k} \in \Lambda-\Lambda}$ p.d. on $\Lambda$, there exists a positive measure $\mu \in \mathbf{M}\left(\mathbf{T}^{n}\right)$ such that

$$
c_{\mathbf{k}}=\hat{\mu}(\mathbf{k})=\frac{1}{(2 \pi)^{n}} \int_{\mathbf{T}^{n}} \mathbf{z}^{-\mathbf{k}} d \mu, \quad \mathbf{k} \in \Lambda-\Lambda .
$$

It is well-known that the set $\{0,1, \ldots, N\} \subset \mathbf{Z}$ has the extension property (see $[\mathrm{Ru}])$. On the other hand, not all sets have the extension property. A simple one-dimensional example is the set $\Lambda=\{0,1,3\}$. Indeed, note that $\Lambda-\Lambda=$ $\{-3,-2,-1,0,1,2,3\}$ and that the condition for a collection of complex numbers $\left\{c_{k}\right\}_{|k| \leq 3}$ to be p.d. on $\Lambda$ is that the Toeplitz matrix

$$
\left(\begin{array}{ccc}
c_{0} & c_{-1} & c_{-3} \\
c_{1} & c_{0} & c_{-2} \\
c_{3} & c_{2} & c_{0}
\end{array}\right)
$$

be positive semi-definite (in the usual sense) while the condition for that same collection to be the restriction of the Fourier coefficients of some positive measure 
on $\mathbf{T}$ to $\Lambda-\Lambda$ is that the Toeplitz matrix

$$
\left(\begin{array}{cccc}
c_{0} & c_{-1} & c_{-2} & c_{-3} \\
c_{1} & c_{0} & c_{-1} & c_{-2} \\
c_{2} & c_{1} & c_{0} & c_{-1} \\
c_{3} & c_{2} & c_{1} & c_{0}
\end{array}\right)
$$

be positive semi-definite, since the extendability of $\left\{c_{k}\right\}_{|k| \leq 3}$ implies that it must also be p.d. on $\{0,1,2,3\}$ and this is in turn equivalent to the stated condition because $\{0,1,2,3\}$ does have the extension property. In general, the first condition is weaker than the second. For example, if we choose $c_{0}=1, c_{1}=0, c_{2}=y, c_{3}=$ $x, c_{-1}=0, c_{-2}=\bar{y}, c_{-3}=\bar{x}$, the fact that the collection $\left\{c_{k}\right\}_{|k| \leq 3}$ is p.d. is equivalent to the inequality $|x|^{2}+|y|^{2} \leq 1$ while the extendability property of that same collection is equivalent to the stronger condition $|x|+|y|^{2} \leq 1$, as can be easily shown. A necessary and sufficient condition for a finite set $\Lambda \in \mathbf{Z}^{n}$ to have the extension property was found by W. Rudin in $[\mathrm{Ru}]$ and, independently, by C. Calderón and R. Pepinsky in [CP].

Theorem 2.2. A finite set $\Lambda \subset \mathbf{Z}^{n}$ has the extension property if and only if for any polynomial $P \in \Pi_{\Lambda-\Lambda}$ satisfying $P(\mathbf{z}) \geq 0$, for all $\mathbf{z} \in \mathbf{T}^{n}$, one can find polynomials $P_{1}, \ldots, P_{m} \in \Pi_{\Lambda}$ such that

$$
P=\sum_{i=1}^{m}\left|P_{i}\right|^{2}
$$

Using this characterization of the extension property as well as a theorem of Hilbert about certain polynomials not representable as sums of squares, Rudin and, independently, Calderón and Pepinsky, were able to obtain examples of very simple higher dimensional sets for which the extension property fails. We need the following definition.

Definition 2.3. If $p_{1}, \ldots, p_{n}$ are non-negative integers, we denote by $R\left(p_{1}, \ldots, p_{n}\right)$ the subset of $\mathbf{Z}^{n}$ defined by $\left\{\left(k_{1}, \ldots, k_{n}\right), k_{i} \in \mathbf{Z}, 0 \leq k_{i} \leq p_{i}, 0 \leq i \leq n\right\}$.

Theorem 2.4 (See $[\mathrm{Ru}]$, [CP]). If $m \geq 3$ and $\Lambda=R(m, m)$, then $\Lambda$ fails to have the extension property.

Later on, L. A. Sakhnovich [Sa], using matricial methods, constructed explicit examples showing that the sets $R(2,2)$ and $R(1,1,1)$ also fail to satisfy the extension property. Moreover, as mentioned in [Sa], the set $R(1,2)$ does have the extension property. However, the methods used in [Sa] do not seem to be easily applicable for more general sets.

It is worth noticing that, although Theorem 2.2 provides a complete characterization of the extension property, the condition stated there is extremely difficult to verify in practice, except for some special cases, even in dimension one. Moreover, it does not provide a way for constructing explicit collections which are not extendable. It is, therefore, of interest to find conditions which are equivalent to the extension property or which imply either the validity of the extension property or its failure for particular classes of sets.

In this paper, we will introduce a new approach to this problem based on orthonormal bases and tight frames of evaluation polynomials, a technique which has been already successfully applied to various moment problems (see, for example, [Ga1], [La1], [La2]). 
We show, in particular, that if a set satisfies the extension property, then it must also satisfy a much stronger property, namely that, for any collection which is s.p.d. on $\Lambda$, there must exist a corresponding representing measure which is supported on set of cardinality equal to $|\Lambda|$, or, equivalently, there must exist an orthogonal basis of evaluation polynomials for the Hilbert space norm naturally associated with the given collection and defined on $\Pi_{\Lambda}$. A similar property must also hold for collections that are p.d., but not s.p.d., on $\Lambda$ (see Theorem 3.9 for a precise statement). This approach yields, in particular, a very elementary algebraic criterion implying the failure of the extension property which can be used in many cases, in particular in all the known examples mentioned above where the extension property fails. Furthermore, that criterion can be used to characterize all the sets satisfying the extension property in the one-dimensional case: it turns out that these sets are precisely the finite arithmetic progressions (see Theorem 4.8). This criterion essentially says that, if the extension property holds for $\Lambda$, then linearly independent polynomials in $\Pi_{\Lambda}$ cannot have many common zeros (on $\mathbf{T}^{n}$ ) if they only have finitely many common zeros. More precisely, under the assumption that the subgroup of $\mathbf{Z}^{n}$ generated by the difference set $\Lambda-\Lambda$ is actually all of $\mathbf{Z}^{n}$, a situation which we can always reduce the problem to, the existence of $k$ linearly independent polynomials in $\Pi_{\Lambda}$ whose set of common zeros on $\mathbf{T}^{n}$ is finite, but of cardinality greater than the number $|\Lambda|-k$, implies the failure of the extension property for $\Lambda$ (Theorem 4.7).

Although this criterion proved itself to be very effective for showing that a set fails the extension property, we do not know, except in the one-dimensional case, if it is actually equivalent to the failure of the extension property. Furthermore, it does not produce explicit counterexamples. Thus, in order to obtain positive results, i.e. to construct sets that satisfy the extension property, and in order to establish a procedure to produce explicit counterexamples in the cases of failure of the extension property, we follow an alternate approach and introduce a condition equivalent to the extension property based on commuting $n$-tuples of unitary matrices (see Theorem 5.2). Let us describe briefly this condition: suppose that, to each point $\mathbf{k} \in \Lambda$, we assign a unit vector $\mathbf{u}_{\mathbf{k}} \in \mathbf{C}^{N}$, where $N<|\Lambda|$, in such a way that the inner product $\mathbf{u}_{\mathbf{k}} \cdot \mathbf{u}_{\mathbf{l}}$ is a function of the difference $\mathbf{k}-\mathbf{l}$ only. It turns out that there is a correspondence, which is one-to-one up to unitary equivalences, between such configurations of unit vectors and collections that are p.d., but not s.p.d., on $\Lambda$. The extension property is then equivalent to the existence, for each one of these configurations, of a commuting $n$-tuple $\left(U_{1}, \ldots, U_{n}\right)$ of $N \times N$ unitary matrices and of a unit vector $\mathbf{x} \in \mathbf{C}^{N}$ with the property that $U_{1}^{k_{1}} U_{2}^{k_{2}} \ldots U_{n}^{k_{n}} \mathbf{x}=\mathbf{u}_{\mathbf{k}}$, for every $\mathbf{k}=\left(k_{1}, \ldots, k_{n}\right) \in \Lambda$. Equivalently, it must always be possible to extend the family $\left\{\mathbf{u}_{\mathbf{k}}\right\}_{\mathbf{k} \in \Lambda}$ to a family $\left\{\mathbf{u}_{\mathbf{k}}\right\}_{\mathbf{k} \in \mathbf{Z}^{n}}$ having again the property that the inner product between any two elements $\mathbf{u}_{\mathbf{k}}$ and $\mathbf{u}_{\mathbf{l}}$ is a function of the difference $\mathbf{k}-\mathbf{l}$ only. This condition can be used to obtain positive results and, in particular, to produce new two-dimensional examples of sets $\Lambda$ satisfying the extension property, such as the sets $\Lambda=R(1, p)$, with $p \geq 1$ (Theorem 5.10). Furthermore, this condition can be combined with the results of section 3 to provide explicit and particularly simple examples of non-extendable collections in the cases of $\Lambda=R(p, q)$ with $p, q \geq 2$ (Theorem 6.1) and $\Lambda=R(p, q, r)$ with $p, q, r \geq 1$ (Theorem 6.3). The idea behind all these counterexamples is that, if a set $\Lambda$ does not satisfy the extension property, then it is usually not difficult, just by trial and error, to produce a configuration of unit vectors on $\Lambda$, as above, that cannot be associated with any commuting $n$-tuple 
of unitary matrices in the sense described above (see Example 5.3). For example, in the case of $\Lambda=R(p, q)$ with $p, q \geq 2$, one can choose an orthonormal basis of $\mathbf{C}^{N}$, where $N=|\Lambda|-2$, place each one of those vectors at exactly one point of the set $\Lambda \backslash\{(p, q),(0, q)\}$ and choose the unit vectors placed at $(p, q)$ and $(0, q)$ to be the same as the one placed at $(0,0)$ and $(p, 0)$, respectively. The condition imposed on the inner products is easily verified and this special configuration corresponds to the collection $\left\{r_{\mathbf{k}}\right\}_{\mathbf{k} \in \Lambda}$ which is p.d. on $\Lambda$ and appears in the statement of Theorem 6.1. Although the collection $\left\{r_{\mathbf{k}}\right\}_{\mathbf{k} \in \Lambda}$ is itself extendable (see Remark 6.2), it fails to satisfy the condition (b) of Theorem 3.9. A counterexample is then easily obtained by following the steps of the proof that (a) implies (b) in Theorem 3.9, which leads to the collection $\left\{c_{\mathbf{k}}\right\}_{\mathbf{k} \in \Lambda}$ in Theorem 6.1 .

The paper is organized as follows. In section 3 , we introduce the notion of evaluation polynomials and tight frames and explain their relationship with truncated trigonometric moment problems. We also prove a result of independent interest which characterizes the extreme points of the set of representing measures associated with a given collection p.d. on $\Lambda$ (Theorem 3.4) as well as Theorem 3.9 that we mentioned above. Section 4 is devoted to proving that the condition on the zeros of polynomials in $\Pi_{\Lambda}$ that we introduced earlier implies the failure of the extension property. That condition is shown to solve the problem completely in the one-dimensional case and is used to produce a vast class of examples of sets, including the known ones, that fail the extension property in higher dimensions. In section 5, we introduce the part of the theory dealing with unitary matrices and show how it can be used to prove both classical and new results about sets that do or do not satisfy the extension property. Finally, the two- and three-dimensional explicit examples of non-extendable collections that we alluded to earlier are given in section 6 .

\section{Weighted Tight FRAMES AND ORTHONORMAL BASES OF EVALUATION POLYNOMIALS}

The notion of frame was introduced by Duffin and Schaeffer in [DS] to generalize the concept of an orthonormal basis in a Hilbert space. It is very useful in the context of non-harmonic Fourier series and wavelet theory (see [Be], [Da], [DGM], [Ga1]). We will restrict the following discussion to the finite-dimensional case.

Definition 3.1. Consider a possibly degenerate inner product denoted by (.,.) and defined on a finite-dimensional complex vector space $\mathcal{P}$. A collection $\left\{e_{n}\right\}$ of vectors in $\mathcal{P}$ is called a frame if there exist constants $C_{1}, C_{2}>0$, called the frame constants, such that

$$
\forall f \in \mathcal{P}, \quad C_{1}\|f\|^{2} \leq \sum_{n}\left|\left(f, e_{n}\right)\right|^{2} \leq C_{2}\|f\|^{2},
$$

where $\|f\|^{2}=(f, f)$ for all $f \in \mathcal{P}$. The frame is called tight if $C_{1}=C_{2}$.

Tight frames are very useful since one can expand elements of $\mathcal{H}=\mathcal{P} / \mathcal{N}$, where $\mathcal{N}=\{f \in \mathcal{H},\|f\|=0\}$ in terms of the elements of a tight frame in pretty much the same way as one would do with orthonormal bases. More explicitly, if $\left\{e_{n}\right\}$ is a tight frame, with frame constants $C_{1}=C_{2}=1$ for simplicity, consider the operator $T$ defined on $\mathcal{H}$, by $T \tilde{f}=\sum\left(f, e_{n}\right) \tilde{e}_{n}$, where $\tilde{f}=f+\mathcal{N}$ for all $f \in \mathcal{P}$. Note that $T$ is well defined on $\mathcal{P} / \mathcal{N}$ since $T=0$ on $\mathcal{N}$ by the definition of a frame. We have 
that, for all $f \in \mathcal{P}$,

$$
(T \tilde{f}, \tilde{f})=\sum\left(f, e_{n}\right)\left(e_{n}, f\right)=\sum\left|\left(f, e_{n}\right)\right|^{2}=\|f\|^{2}=\|\tilde{f}\|^{2},
$$

and thus $T$ must be the identity operator, i.e. $\tilde{f}=\sum\left(f, e_{n}\right) \tilde{e}_{n}$, for all $f \in \mathcal{P}$, which is the usual expansion formula in terms of an orthonormal basis. Let us emphasize that, in general, there are more vectors in a frame that we need to expand elements of $\mathcal{P} / \mathcal{N}$ with. In particular, the number of elements of a frame is larger than the dimension of $\mathcal{P} / \mathcal{N}$ unless the collection $\left\{\tilde{e}_{n}\right\}$ actually forms a basis of $\mathcal{P} / \mathcal{N}$.

Given a collection $\left\{c_{\mathbf{k}}\right\}_{\mathbf{k} \in \Lambda-\Lambda}$ which is s.p.d. on $\Lambda$, one can define a natural inner product on $\Pi_{\Lambda}$ which turns it into a finite-dimensional Hilbert space denoted by $\mathcal{H}$. If $P(\mathbf{z})=\sum_{\mathbf{k} \in \Lambda} a_{\mathbf{k}} \mathbf{z}^{\mathbf{k}}$ and $Q(\mathbf{z})=\sum_{\mathbf{l} \in \Lambda} b_{\mathbf{l}} \mathbf{z}^{\mathbf{l}}$ belong to $\Pi_{\Lambda}$, we simply let

$$
[P, Q]=\sum_{\mathbf{k}, \mathbf{l} \in \Lambda} c_{\mathbf{l}-\mathbf{k}} a_{\mathbf{k}} \overline{b_{\mathbf{l}}}
$$

Since our collection $\left\{c_{\mathbf{k}}\right\}_{\mathbf{k} \in \Lambda-\Lambda}$ is s.p.d., the associated semi-norm defined by $\|P\|=$ $[P, P]^{1 / 2}$ for $P \in \Pi_{\Lambda}$ is actually a norm. If the collection $\left\{c_{\mathbf{k}}\right\}_{\mathbf{k} \in \Lambda-\Lambda}$ is p.d. but not s.p.d. on $\Lambda$, there will exist non-zero polynomials $P$ in $\Pi_{\Lambda}$ satisfying $\|P\|=0$, but a norm can still be defined on the quotient space $\Pi_{\Lambda} / \mathcal{N}$, where $\mathcal{N}=\{P \in$ $\left.\Pi_{\Lambda},\|P\|=0\right\}$ and we define $\mathcal{H}=\Pi_{\Lambda} / \mathcal{N}$. A very important notion associated with a given inner product as defined in (3.1) is that of an evaluation polynomial.

Definition 3.2. Given a collection $\left\{c_{\mathbf{k}}\right\}_{\mathbf{k} \in \Lambda-\Lambda}$ which is s.p.d. on $\Lambda$ and $\alpha \in \mathbf{T}^{n}$, we define $E_{\alpha}$, the evaluation polynomial at $\alpha$, to be the unique polynomial in $\Pi_{\Lambda}$ satisfying $P(\alpha)=\left[P, E_{\alpha}\right]$, for all $P \in \Pi_{\Lambda}$, where the inner product [.,.] is defined in (3.1). If the collection $\left\{c_{\mathbf{k}}\right\}_{\mathbf{k} \in \Lambda-\Lambda}$ is p.d. but not s.d.p. on $\Lambda$, we let

$$
\begin{aligned}
\Omega & =\left\{\alpha \in \mathbf{T}^{n}, P(\alpha)=0, \forall P \in \mathcal{N}\right\} \\
& =\left\{\alpha \in \mathbf{T}^{n}, \exists C_{\alpha}>0,|P(\alpha)| \leq C_{\alpha}\|P\|, \forall P \in \Pi_{\Lambda}\right\},
\end{aligned}
$$

and if $\alpha \in \Omega$, we let $E_{\alpha}$ be any polynomial satisfying $P(\alpha)=\left[P, E_{\alpha}\right]$ for all $P \in \Pi_{\Lambda}$. In that case, the evaluation polynomials are unique up to the addition of any element of $\mathcal{N}$.

We note that the existence and the uniqueness of $E_{\alpha}$ up to an element of $\mathcal{N}$ are guaranteed by the Riesz representation theorem applied in $\mathcal{H}$. Evaluation polynomials have been used successfully in many situations where moment problems are involved (see [Ga1], [Ga2], [La1], [La2]). Their importance in the present context stems from the following result.

Proposition 3.3. Let $\left\{c_{\mathbf{k}}\right\}_{\mathbf{k} \in \Lambda-\Lambda}$ be a collection which is p.d. and consider the possibly degenerate inner product defined on $\Pi_{\Lambda}$ by (3.1). Given numbers $d_{1}, \ldots, d_{m}$ $>0$ and $\alpha_{1}, \ldots, \alpha_{m} \in \mathbf{T}^{n}$, a necessary and sufficient condition for the collection $\left\{\sqrt{d_{i}} E_{\alpha_{i}}\right\}_{i=1}^{m}$ to be a tight frame with frame constant 1 is that the measure $\mu=$ $(2 \pi)^{n} \sum_{i=1}^{m} d_{i} \delta_{\alpha_{i}}$ be a representing measure associated with $\left\{c_{\mathbf{k}}\right\}_{\mathbf{k} \in \Lambda-\Lambda}$, where $\delta_{\alpha_{i}}$ denotes the Dirac mass at $\alpha_{i}$.

Proof. If the collection $\left\{\sqrt{d_{i}} E_{\alpha_{i}}\right\}_{i=1}^{m}$ forms a tight frame, then, for all $P \in \Pi_{\Lambda}$, we have that

$$
\|P\|^{2}=\sum_{i=1}^{m}\left|\left[P, \sqrt{d_{i}} E_{\alpha_{i}}\right]\right|^{2},
$$


or, equivalently, that

$$
\|P\|^{2}=\sum_{i=1}^{m} d_{i}\left|P\left(\alpha_{i}\right)\right|^{2}=\frac{1}{(2 \pi)^{n}} \int_{\mathbf{T}^{n}}|P|^{2} d \mu .
$$

This implies, for all $P, Q \in \Pi_{\Lambda}$, the identity

$$
[P, Q]=\frac{1}{(2 \pi)^{n}} \int_{\mathbf{T}^{n}} P \bar{Q} d \mu .
$$

In particular, choosing $P(\mathbf{z})=\mathbf{z}^{\mathbf{k}}$ and $Q(\mathbf{z})=\mathbf{z}^{\mathbf{l}}$, where $\mathbf{k}, \mathbf{l} \in \Lambda$, we obtain that, for all $\mathbf{k}, \mathbf{l} \in \Lambda$,

$$
c_{\mathbf{1 - k}}=\frac{1}{(2 \pi)^{n}} \int_{\mathbf{T}^{n}} \mathbf{z}^{\mathbf{k}-\mathbf{l}} d \mu,
$$

i.e., that $\mu$ is a representing measure associated with $\left\{c_{\mathbf{k}}\right\}_{\mathbf{k} \in \Lambda-\Lambda}$. The converse is immediate.

A collection of polynomials in $\Pi_{\Lambda}$, as in the previous proposition, will be called a weighted tight frame of evaluation polynomials. Thus, the representing measures with finite support associated with a collection $\left\{c_{\mathbf{k}}\right\}_{\mathbf{k} \in \Lambda-\Lambda}$ p.d. on $\Lambda$ are in oneto-one correspondence with the weighted tight frames of evaluation polynomials associated with that same collection. One can ask the question as to whether or not the existence of a representing measure for a given collection $\left\{c_{\mathbf{k}}\right\}_{\mathbf{k} \in \Lambda-\Lambda}$ p.d. on $\Lambda$ implies the existence of a representing measure with finite support associated with that same collection. An affirmative answer to that question is given in the next result, where the extreme points of the set of representing measures associated with a given collection are characterized. This is an analogue of a result due to Naimark in the context of the classical moment problem (cf. [AK, p. 47]). Note that, if the set of extreme points is non-empty, it is clearly convex and weak-* compact, and thus equal to the closure of the convex-hull of its extreme points by the Krein-Milman theorem.

Theorem 3.4. Let $A$ be the set of representing measures associated with a collection $\left\{c_{\mathbf{k}}\right\}_{\mathbf{k} \in \Lambda-\Lambda}$ p.d. on $\Lambda$. Then, $\mu$ is an extreme point of $A$ if and only if $\Pi_{\Lambda-\Lambda}$ is dense in $L^{1}(\mu)$. In this case, in particular, the cardinality of the support of $\mu$ is at most $|\Lambda-\Lambda|$.

Proof. Suppose that $\mu$ is an extreme point of $A$ and that $\Pi_{\Lambda-\Lambda}$ is not dense in $L^{1}(\mu)$. Then, using the Hahn-Banach theorem, we can find $g \in L^{\infty}(\mu)$ such that $g \neq 0$ and $\int_{\mathbf{T}^{n}} P g d \mu=0$, for all $P \in \Pi_{\Lambda-\Lambda}$. Since $\bar{P} \in \Pi_{\Lambda-\Lambda}$ when $P \in \Pi_{\Lambda-\Lambda}$, we can assume that $g$ is real and also that $\|g\|_{\infty} \leq 1 / 2$. Letting $\mu_{1}=(1+g) \mu$ and $\mu_{2}=(1-g) \mu$, we see that both $\mu_{1}$ and $\mu_{2}$ are positive measures and if $P \in \Pi_{\Lambda-\Lambda}$, we have that

$$
\int_{\mathbf{T}^{n}} P d \mu_{1}=\int_{\mathbf{T}^{n}} P d \mu_{2}=\int_{\mathbf{T}^{n}} P d \mu
$$

which shows that both $\mu_{1}$ and $\mu_{2}$ are representing measures. Since $\mu=\frac{1}{2} \mu_{1}+\frac{1}{2} \mu_{2}$, and $\mu$ is an extreme point, we deduce that $\mu=\mu_{1}=\mu_{2}$, or, equivalently, that $g=0$ a.e. $d \mu$ which yields a contradiction. Hence, $\Pi_{\Lambda-\Lambda}$ is dense in $L^{1}(\mu)$. Conversely, if $\Pi_{\Lambda-\Lambda}$ is dense in $L^{1}(\mu)$, the dimension of $L^{1}(\mu)$ is at most $|\Lambda-\Lambda|$, the dimension of $\Pi_{\Lambda-\Lambda}$, and this easily implies that $\mu$ is a weighted sum of at most $|\Lambda-\Lambda|$ Dirac masses. If $\mu=\frac{1}{2} \mu_{1}+\frac{1}{2} \mu_{2}$, where $\mu_{1}, \mu_{2} \in A$, it follows that the support of $\mu_{i}$ is 
contained in that of $\mu$ for $i=1,2$. Now, if $\alpha$ belongs to the support of $\mu$, we can find, using the density of $\Pi_{\Lambda-\Lambda}$ in $L^{1}(\mu)$, an element $P \in \Pi_{\Lambda-\Lambda}$ such that $P(\alpha)=1$ and $P(\beta)=0$, for all $\beta$ belonging to the support of $\mu$, with $\beta \neq \alpha$. We deduce, therefore, that

$$
\mu(\{\alpha\})=\int_{\mathbf{T}^{n}} P d \mu=\int_{\mathbf{T}^{n}} P d \mu_{i}=\mu_{i}(\{\alpha\}),
$$

for $i=1,2$, which implies that $\mu=\mu_{1}=\mu_{2}$, i.e. $\mu$ is an extreme point of $A$.

It follows immediately from the previous theorem that if $\Lambda$ has the extension property, then weighted tight frames of evaluation polynomials can always be associated with any collection which is p.d. on $\Lambda$. A natural question to ask is whether or not one can always find, in that case, a collection of the form $\left.\left\{E_{\beta} / \| E_{\beta}\right\} \|\right\}_{\beta \in A}$, where $A$ is some finite subset of $\mathbf{T}^{n}$, forming an orthonormal basis associated with that collection. Since we may be dealing with a degenerate inner product, we first need to clarify the notion of orthonormal basis in that case.

Definition 3.5. Let us consider a possibly degenerate inner product (.,.) defined on a finite-dimensional complex vector space $\mathcal{P}$. We say that a collection $\left\{e_{n}\right\}$ is an orthonormal basis for $\mathcal{P}$ with respect to the inner product (.,.) if the collection $\left\{\tilde{e}_{n}\right\}$ is an orthonormal basis in the usual sense in the quotient space $\mathcal{P} / \mathcal{N}$, where $\mathcal{N}=\{f \in \mathcal{P},\|f\|=0\}$ and $\tilde{f}=f+\mathcal{N}$ for all $f \in \mathcal{P}$.

Lemma 3.6. The collection $\left\{e_{n}\right\}$ is an orthonormal basis for $\mathcal{P}$ with respect to the inner product (.,.) if and only if, for all $f \in \mathcal{P},\|f\|^{2}=\sum\left|\left(f, e_{n}\right)\right|^{2}$ and the mapping $T$ from $\mathcal{P}$ to $\ell^{2}, f \mapsto\left\{\left(f, e_{n}\right)\right\}$, is onto.

Proof. If $\left\{e_{n}\right\}$ is an orthonormal basis for $\mathcal{P}$ with respect to the inner product (.,.), it follows that, for all $f \in \mathcal{P}$,

$$
\|f\|^{2}=\|\tilde{f}\|^{2}=\sum\left|\left(\tilde{f}, \tilde{e}_{n}\right)\right|^{2}=\sum\left|\left(f, e_{n}\right)\right|^{2} .
$$

Furthermore, if $T$ wasn't onto, there would exist $\left\{a_{n}\right\} \in \ell^{2}$ such that $\sum\left(f, e_{n}\right) \overline{a_{n}}=$ 0 , for all $f \in \mathcal{P}$ which would imply that $\sum\left(\tilde{f}, \tilde{e}_{n}\right) \overline{a_{n}}=0$, for all such $f$, and thus that all the $a_{n}$ 's are zero, since $\left\{\tilde{e}_{n}\right\}$ is an orthonormal basis for $\mathcal{P} / \mathcal{N}$. Conversely, if $\|f\|^{2}=\sum\left|\left(f, e_{n}\right)\right|^{2}$ for all $f \in \mathcal{P}$ and $T$ is onto, then $\|\tilde{f}\|^{2}=\sum\left|\left(\tilde{f}, \tilde{e}_{n}\right)\right|^{2}$ for all $f \in \mathcal{P}$, and thus the collection $\left\{\tilde{e}_{n}\right\}$ forms a tight frame in $\mathcal{P} / \mathcal{N}$. In particular, $\tilde{f}=\sum\left(\tilde{f}, \tilde{e}_{n}\right) \tilde{e}_{n}$ for all $f \in \mathcal{P}$ and thus $\left\{\tilde{e}_{n}\right\}$ is complete. To show the orthonormality of the $\tilde{e}_{n}$ 's, we notice that $\tilde{e}_{k}=\sum_{n}\left(\tilde{e}_{k}, \tilde{e}_{n}\right) \tilde{e}_{n}$ and, thus, for all $f \in \mathcal{P}, \sum_{n}\left\{\left(\tilde{e}_{k}, \tilde{e}_{n}\right)-\delta_{k n}\right\}\left(\tilde{f}, \tilde{e}_{n}\right)=0$, where $\delta_{k n}=1$ if $k=n$ and 0 otherwise. Since the mapping $T$ is onto, it follows that $\left(\tilde{e}_{k}, \tilde{e}_{n}\right)=\delta_{k n}$, proving the desired orthonormality.

Definition 3.7. If $\alpha \in \mathbf{T}^{n}$, we denote by $L_{\alpha}$ the linear form on $\Pi_{\Lambda}$ defined by $L_{\alpha}(P)=P(\alpha)$, for $P \in \Pi_{\Lambda}$.

The following lemma is an immediate consequence of Lemma 3.6.

Lemma 3.8. Given a collection $\left\{c_{\mathbf{k}}\right\}_{\mathbf{k} \in \Lambda-\Lambda}$ p.d. on $\Lambda$, let us consider the associated set $\Omega=\left\{\alpha \in \mathbf{T}^{n}, P(\alpha)=0, \forall P \in \mathcal{N}\right\}$, where $\mathcal{N}=\left\{P \in \Pi_{\Lambda},\|P\|=0\right\}$ and $\|$.$\| denotes the semi-norm associated with \left\{c_{\mathbf{k}}\right\}_{\mathbf{k} \in \Lambda-\Lambda}$. Then, a set of polynomials of the form $\left\{E_{\alpha} /\left\|E_{\alpha}\right\|\right\}_{\alpha \in A}$, where $A$ is a finite subset of $\Omega$, is an orthonormal basis for $\Pi_{\Lambda}$ with respect to the inner product associated with $\left\{c_{\mathbf{k}}\right\}_{\mathbf{k} \in \Lambda-\Lambda}$ if and 
only if $\|P\|^{2}=\sum_{\alpha \in A}|P(\alpha)|^{2} /\left\|E_{\alpha}\right\|^{2}$ for all $P \in \Pi_{\Lambda}$ and, in this case, the family of linear forms $\left\{L_{\alpha}\right\}_{\alpha \in A}$ is linearly independent.

We can now answer the question raised above about the connection between sets having the extension property and the existence of orthonormal bases of normalized evaluation polynomials.

Theorem 3.9. The following statements are equivalent.

(a) The finite set $\Lambda \subset \mathbf{Z}^{n}$ has the extension property.

(b) For any collection $\left\{c_{\mathbf{k}}\right\}_{\mathbf{k} \in \Lambda-\Lambda}$ p.d. on $\Lambda$, the set $\Omega=\left\{\beta \in \mathbf{T}^{n}, P(\beta)=\right.$ $0, \forall P \in \mathcal{N}\}$, where $\mathcal{N}=\left\{P \in \Pi_{\Lambda},\|P\|=0\right\}$, and $\|$.$\| denotes the semi-norm$ associated with $\left\{c_{\mathbf{k}}\right\}_{\mathbf{k} \in \Lambda-\Lambda}$, is non-empty, and, for any $\alpha \in \Omega$, there exists a finite set $B \subset \Omega$ containing $\alpha$ such that the family $\left\{E_{\beta} /\left\|E_{\beta}\right\|\right\}_{\beta \in B}$ forms an orthonormal basis for $\Pi_{\Lambda}$ with respect to the inner product associated with $\left\{c_{\mathbf{k}}\right\}_{\mathbf{k} \in \Lambda-\Lambda}$.

Proof. It is clear that (b) implies (a) since, if $\left\{E_{\beta} /\left\|E_{\beta}\right\|\right\}_{\beta \in B}$ is any orthonormal basis for $\Pi_{\Lambda}$ with respect to the inner product associated with a collection $\left\{c_{\mathbf{k}}\right\}_{\mathbf{k} \in \Lambda-\Lambda}$ p.d. on $\Lambda$, we have, using Lemma 3.6, that, for all $P \in \Pi_{\Lambda}$,

$$
\|P\|^{2}=\sum_{\beta \in B}|P(\beta)|^{2} /\left\|E_{\beta}\right\|^{2}=\frac{1}{(2 \pi)^{n}} \int_{\mathbf{T}^{n}}|P|^{2} d \mu
$$

where $\mu$ is the measure $(2 \pi)^{n} \sum_{\beta \in B}\left\|E_{\beta}\right\|^{-2} \delta_{\beta}$ and $\delta_{\beta}$ denotes the Dirac mass at $\beta$. This implies that $c_{\mathbf{k}}=\frac{1}{(2 \pi)^{n}} \int_{\mathbf{T}^{n}} \mathbf{z}^{-\mathbf{k}} d \mu$, for all $\mathbf{k} \in \Lambda-\Lambda$, proving (a). Conversely, assuming that (a) holds, let $\left\{c_{\mathbf{k}}\right\}_{\mathbf{k} \in \Lambda-\Lambda}$ be a collection p.d. on $\Lambda$ and consider the associated set $\Omega \subset \mathbf{T}^{n}$. Note first that $\Omega \neq \emptyset$, since any representing measure associated with the collection $\left\{c_{\mathbf{k}}\right\}_{\mathbf{k} \in \Lambda-\Lambda}$ must be supported in $\Omega$. We need to show that, given $\alpha_{1} \in \Omega$, there exist $\alpha_{2}, \ldots, \alpha_{m} \in \Omega$, where $m=\operatorname{dim}\left(\Pi_{\Lambda} / \mathcal{N}\right)=$ $|\Lambda|-\operatorname{dim}(\mathcal{N})$, such that $\left[E_{\alpha_{i}}, E_{\alpha_{j}}\right]=E_{\alpha_{i}}\left(\alpha_{j}\right)=0$, for all $1 \leq i, j \leq m$, with $i \neq j$. We will show the existence of $\alpha_{2}, \ldots, \alpha_{m}$ by induction. If $E_{\alpha_{1}}, \ldots, E_{\alpha_{k}}$, where $1 \leq k \leq m-1$, have been constructed in such a way that $E_{\alpha_{i}}\left(\alpha_{j}\right)=0$, for all $1 \leq i, j \leq k$, with $i \neq j$, we claim that, for all $P \in \Pi_{\Lambda}$, we have the inequality

$$
\|P\|^{2}-\sum_{i=1}^{k}\left\|E_{\alpha_{i}}\right\|^{-2}\left|P\left(\alpha_{i}\right)\right|^{2} \geq 0 .
$$

Indeed, writing $P=\left\{\sum_{i=1}^{k}\left[P, E_{\alpha_{i}}\right] E_{\alpha_{i}} /\left\|E_{\alpha_{i}}\right\|^{2}\right\}-Q$, we have $\left[Q, E_{\alpha_{i}}\right]=0$, for $i=1, \ldots, k$, and thus,

$$
\|P\|^{2}=\left\|Q+\sum_{i=1}^{k} P\left(\alpha_{i}\right)\right\| E_{\alpha_{i}}\left\|^{-2} E_{\alpha_{i}}\right\|^{2}=\|Q\|^{2}+\sum_{i=1}^{k}\left\|E_{\alpha_{i}}\right\|^{-2}\left|P\left(\alpha_{i}\right)\right|^{2} .
$$

Writing $P=\sum_{\mathbf{k} \in \Lambda} a_{\mathbf{k}} \mathbf{z}^{\mathbf{k}}$, it is easily checked that the left-hand side of (3.2) is equal to the expression

$$
\sum_{\mathbf{k}, \mathbf{l} \in \Lambda} s_{\mathbf{l}-\mathbf{k}} a_{\mathbf{k}} \overline{a_{\mathbf{l}}}
$$


where $s_{\mathbf{j}}=c_{\mathbf{j}}-\sum_{i=1}^{k}\left\|E_{\alpha_{i}}\right\|^{-2} \alpha_{i}{ }^{-\mathbf{j}}$, for all $\mathbf{j} \in \Lambda-\Lambda$. Since $\Lambda$ is assumed to have the extension property, there exists a positive measure $\nu \in \mathbf{M}\left(\mathbf{T}^{n}\right)$ satisfying

$$
\|P\|^{2}-\sum_{i=1}^{k}\left\|E_{\alpha_{i}}\right\|^{-2}\left|P\left(\alpha_{i}\right)\right|^{2}=\frac{1}{(2 \pi)^{n}} \int_{\mathbf{T}^{n}}|P|^{2} d \nu,
$$

for all $P \in \Pi_{\Lambda}$. In particular, replacing $P$ in (3.3) by $E_{\alpha_{j}}$, where $1 \leq j \leq k$, we obtain that

$$
\int_{\mathbf{T}^{n}}\left|E_{\alpha_{j}}\right|^{2} d \nu=0
$$

for each such $j$. This shows that the support of $\nu$ must be contained in the intersection $\bigcap_{i=1}^{k}\left\{\beta \in \mathbf{T}^{n}, E_{\alpha_{i}}(\beta)=0\right\}$. Furthermore, since

$$
\|P\|^{2}=\int_{\mathbf{T}^{n}}|P|^{2} d \nu=0
$$

for all $P \in \mathcal{N}$, it follows that the support of $\nu$ must also be contained in $\Omega$. Since equality in (3.3) for all $P \in \Pi_{\Lambda}$ is impossible, $k$ being strictly less than $m$, we deduce therefore that the measure $\nu$ cannot be identically zero. Hence, it suffices now to choose any point $\alpha_{k+1}$ in the support of $\nu$ to fulfill our conditions. This concludes the proof.

Remark 3.10. In the particular case where the collection $\left\{c_{\mathbf{k}}\right\}_{\mathbf{k} \in \Lambda-\Lambda}$ is defined by $c_{\mathbf{k}}=\delta_{0 k}$, for $\mathbf{k} \in \Lambda-\Lambda$, the problem of the existence of an orthonormal basis of evaluation polynomials, as in Theorem 3.9, is a discrete version of the "Spectral Pair" problem, introduced by B. Fuglede in [Fu], which asked for conditions under which, for a given measurable set $A \subset \mathbf{R}^{n}$ with finite measure, there exists an associated collection of exponentials $\left\{e_{\lambda_{n}}\right\}$, where $e_{\lambda}(x)=\exp (2 \pi i \lambda . x)$ for $\lambda, x \in$ $\mathbf{R}^{n}$, which, when normalized and restricted to $A$, form a complete orthonormal system for the space $L_{A}^{2}$ of square integrable functions vanishing a.e. outside $A$. Recent results on this problem have been obtained by P. E. T. Jorgensen and S. Pedersen [JP].

Example 3.11. Using Theorem 3.9, one can give an easy proof that the set $\Lambda=$ $\{0,1,3\}$ does not have the extension property. Indeed, if one considers the usual $L^{2}$-norm on $\Pi_{\Lambda}$ (corresponding to $c_{\mathbf{k}}=\delta_{0 k}$, for $\mathbf{k} \in \Lambda-\Lambda$ ), one finds that $E_{1}(z)=1+z+z^{3}$, a polynomial without zeros on the unit circle.

\section{A CONDition implying the failure of the eXtension property}

In this section, we will consider a very useful condition implying the failure of the extension property. We will first need some preliminary definitions and lemmas.

Definition 4.1. We will denote by $G(\Lambda-\Lambda)$ the subgroup of $\mathbf{Z}^{n}$ generated by $\Lambda-\Lambda$.

Lemma 4.2. Given a finite set $\Lambda \subset \mathbf{Z}^{n}$, we have $G(\Lambda-\Lambda)=\mathbf{Z}^{n}$ if and only if, for every $\alpha, \beta \in \mathbf{T}^{n}$, with $\alpha \neq \beta$, there exists a polynomial $P \in \Pi_{\Lambda}$ such that $|P(\alpha)| \neq|P(\beta)|$.

Proof. Given $\alpha, \beta \in \mathbf{T}^{n}$, the fact that $|P(\alpha)|=|P(\beta)|$, for all $P \in \Pi_{\Lambda}$, is equivalent to $\alpha^{\mathbf{k}-\mathbf{l}}=\beta^{\mathbf{k}-\mathbf{1}}$, for all $\mathbf{k}, \mathbf{l} \in \Lambda$. If $G(\Lambda-\Lambda)=\mathbf{Z}^{n}$ and, for some $\alpha, \beta \in \mathbf{T}^{n}$, we had that $|P(\alpha)|=|P(\beta)|$, for all $P \in \Pi_{\Lambda}$, this would imply that $\alpha^{\mathbf{m}}=\beta^{\mathbf{m}}$, for all $\mathbf{m} \in \mathbf{Z}^{n}$, and, thus, that $\alpha=\beta$. If, on the other hand, $G(\Lambda-\Lambda) \neq \mathbf{Z}^{n}$, consider a 
non-trivial character $\gamma$ of the group $\mathbf{Z}^{n} / G(\Lambda-\Lambda)$ and define the character $\tilde{\gamma}$ of the group $\mathbf{Z}^{n}$ by $\tilde{\gamma}(\mathbf{m})=\gamma(\mathbf{m}+G(\Lambda-\Lambda))$, for $\mathbf{m} \in \mathbf{Z}^{n}$. Since $\mathbf{T}^{n}$ is the dual group of $\mathbf{Z}^{n}$, there exists $\alpha \in \mathbf{T}^{n}$, different from the identity, such that $\tilde{\gamma}(\mathbf{m})=\alpha^{\mathbf{m}}$, for all $\mathbf{m} \in \mathbf{Z}^{n}$. In particular, we have that $\alpha^{\mathbf{k}-\mathbf{l}}=1$, for all $\mathbf{k}, \mathbf{l} \in \Lambda$ and thus $|P(\alpha)|=|P(\mathbf{1})|$, for all $P \in \Pi_{\Lambda}$, where $\mathbf{1}$ denotes the identity in $\mathbf{T}^{n}$ and $\alpha \neq \mathbf{1}$. This proves the lemma.

The following lemma shows that the extension property is invariant under translations and one-to-one homomorphisms.

Lemma 4.3. Let $\Phi: \mathbf{Z}^{k} \rightarrow \mathbf{Z}^{k}$ be a translation by some fixed element $\mathbf{m}$ of $\mathbf{Z}^{n}$ or let $\Phi: \mathbf{Z}^{k} \rightarrow \mathbf{Z}^{n}$, where $k \leq n$, be a one-to-one homomorphism. Then, a finite set $\Lambda \subset \mathbf{Z}^{k}$ has the extension property if and only if $\Phi(\Lambda)$ has that same property.

Proof. If $\Phi$ is a translation, the statement is obvious. If $\Phi$ is a one-to-one homomorphism from $\mathbf{Z}^{k}$ to $\mathbf{Z}^{n}$, where $k \leq n$, we denote by $\Phi^{*}: \mathbf{T}^{n} \rightarrow \mathbf{T}^{k}$ the dual automorphism defined by $\left[\Phi^{*}(\alpha)\right]^{\mathbf{k}}=\alpha^{\Phi(\mathbf{k})}$, for $\alpha \in \mathbf{T}^{n}$ and $\mathbf{k} \in \mathbf{Z}^{k}$. It is clear that the transformation from $\Pi_{\Phi(\Lambda)}$ to $\Pi_{\Lambda}$ that sends the polynomial $P(\mathbf{z})=\sum_{\mathbf{k} \in \Lambda} a_{\Phi(\mathbf{k})} \mathbf{z}^{\Phi(\mathbf{k})}$ to the polynomial $\tilde{P}(\mathbf{z})=\sum_{\mathbf{k} \in \Lambda} b_{\mathbf{k}} \mathbf{z}^{\mathbf{k}}$, where $b_{\mathbf{k}}=a_{\Phi(\mathbf{k})}$ for all $\mathbf{k} \in \Lambda$ is a bijective correspondence between $\Pi_{\Phi(\Lambda)}$ and $\Pi_{\Lambda}$. If the collection $\left\{c_{\mathbf{k}}\right\}_{\mathbf{k} \in \Phi(\Lambda)-\Phi(\Lambda)}$ is p.d. on $\Phi(\Lambda)$, the collection $\left\{s_{\mathbf{k}}\right\}_{\mathbf{k} \in \Lambda}$ defined by the formula $s_{\mathbf{k}}=c_{\Phi(\mathbf{k})}$, for $\mathbf{k} \in \Lambda-\Lambda$, is p.d. on $\Lambda$. Indeed, using the injectivity of $\Phi$, we have that

$$
\sum_{\mathbf{k}, \mathbf{l} \in \Lambda} s_{\mathbf{k}-\mathbf{l}} b_{\mathbf{k}} \overline{b_{\mathbf{l}}}=\sum_{\mathbf{k}, \mathbf{l} \in \Lambda} c_{\Phi(\mathbf{k}-\mathbf{l})} b_{\mathbf{k}} \overline{b_{\mathbf{l}}}=\sum_{\mathbf{k}, \mathbf{l} \in \Lambda} c_{\Phi(\mathbf{k})-\Phi(\mathbf{l})} a_{\Phi(\mathbf{k})} \overline{a_{\Phi(\mathbf{l})}} \geq 0 .
$$

If $\Lambda$ has the extension property, there exist, using Lemma 3.8, numbers $d_{1}, \ldots$, $d_{m}>0$ and $\alpha_{1}, \ldots, \alpha_{m} \in \mathbf{T}^{k}$ such that

$$
\sum_{\mathbf{k}, \mathbf{l} \in \Lambda} s_{\mathbf{k}-\mathbf{l}} b_{\mathbf{k}} \overline{b_{\mathbf{l}}}=\sum_{i=1}^{m} d_{i}\left|\sum_{\mathbf{k} \in \Lambda} b_{\mathbf{k}} \alpha_{i}^{\mathbf{k}}\right|^{2} .
$$

We note now that the fact that $\Phi$ is one-to-one is equivalent to the fact that $\Phi^{*}$ is onto. Indeed, the non-surjectivity of $\Phi^{*}$ is equivalent to the existence of a nontrivial character which is identically one on the subgroup $\Phi^{*}\left(\mathbf{T}^{n}\right)$ of $\mathbf{T}^{k}$ and, thus, to the existence of a non-zero element $\mathbf{k}$ of $\mathbf{Z}^{k}$ satisfying $\left[\Phi^{*}(\alpha)\right]^{\mathbf{k}}=1$, or $\alpha^{\Phi(\mathbf{k})}=1$, for all $\alpha \in \mathbf{T}^{n}$. This is, in turn, equivalent $\Phi(\mathbf{k})=0$, for some non-zero $\mathbf{k} \in \mathbf{Z}^{k}$ or to the non-injectivity of $\Phi$. Hence, since $\Phi$ is assumed to be one-to-one, we can find, using the surjectivity of $\Phi^{*}, \beta_{1}, \ldots, \beta_{m} \in \mathbf{T}^{n}$ satisfying $\Phi^{*}\left(\beta_{i}\right)=\alpha_{i}$, for all $i=1, \ldots, m$. Since $\left[\Phi^{*}\left(\beta_{i}\right)\right]^{\mathbf{k}}=\beta_{i}^{\Phi(\mathbf{k})}$, we deduce, therefore, that

$$
\sum_{\mathbf{k}, \mathbf{l} \in \Lambda} c_{\Phi(\mathbf{k})-\Phi(\mathbf{l})} a_{\Phi(\mathbf{k})} \overline{a_{\Phi(\mathbf{l})}}=\sum_{i=1}^{m} d_{i}\left|\sum_{\mathbf{k} \in \Lambda} a_{\Phi(\mathbf{k})} \beta_{i}^{\Phi(\mathbf{k})}\right|^{2}=\sum_{i=1}^{m} d_{i}\left|P\left(\beta_{i}\right)\right|^{2},
$$

which shows that $\Phi(\Lambda)$ also has the extension property. The proof of the converse implication is similar and left to the reader.

Lemma 4.4. Given a finite set $\Lambda \subset \mathbf{Z}^{n}$ with $0 \in \Lambda$, there exist an integer $k$ with $1 \leq k \leq n$, a finite subset $\Delta$ of $\mathbf{Z}^{k}$, and a one-to-one homomorphism $\Phi: \mathbf{Z}^{k} \rightarrow \mathbf{Z}^{n}$ such that $\Phi(\Delta)=\Lambda$ and $G(\Delta-\Delta)=\mathbf{Z}^{k}$. 
Proof. We let $k$ be the number of any collection of linearly independent vectors $\mathbf{m}_{1}, \ldots, \mathbf{m}_{k}$ generating $G(\Lambda-\Lambda)$, i.e. such that

$$
G(\Lambda-\Lambda)=\left\{\sum_{i=1}^{k} p_{i} \mathbf{m}_{i}, p_{1}, \ldots, p_{k} \in \mathbf{Z}\right\}
$$

and we define the homomorphism $\Phi: \mathbf{Z}^{k} \rightarrow \mathbf{Z}^{n}$ by $\Phi\left(\left(p_{1}, \ldots, p_{k}\right)\right)=\sum_{i=1}^{k} p_{i} \mathbf{m}_{i}$. Since the $\mathbf{m}_{i}$ 's are linearly independent, $\Phi$ is clearly one-to-one. We let $\Delta=$ $\Phi^{-1}(\Lambda)$. Clearly, $\Phi(\Delta)=\Lambda$ and $G(\Delta-\Delta)=\mathbf{Z}^{k}$, since $\Phi^{-1}\left(\mathbf{m}_{i}\right)=\mathbf{e}_{i} \in \Delta-\Delta$ for $i=1, \ldots, k$, where $\mathbf{e}_{i}$ denotes the vector in $\mathbf{Z}^{k}$ whose $i$-th component is one and with all other components equal to zero.

Combining the two previous lemmas, we see that, as far as the extension property is concerned, we can restrict ourselves to considering sets $\Lambda \subset \mathbf{Z}^{n}$ having the property that $G(\Lambda-\Lambda)=\mathbf{Z}^{n}$.

Lemma 4.5. Let $\mathbf{x}=\left(x_{1}, \ldots, x_{m}\right) \in \mathbf{C}^{m}$, where $m \geq 2$, and suppose that $\mathbf{x}$ has, at least, two non-zero components. Then, there is no finite set $F \subset \mathbf{C}^{m}$ with the properties that $0 \notin F$ and that for any $d_{1}, \ldots, d_{m}>0$, there exists $\mathbf{y}=$ $\left(y_{1}, \ldots, y_{m}\right) \in F$ satisfying $\sum_{i=1}^{m} d_{i} x_{i} \overline{y_{i}}=0$.

Proof. Let us suppose the contrary. There is no loss of generality in assuming that both $x_{1}$ and $x_{2}$ are non-zero. Let us fix $d_{1}, d_{2}>0$ and let $d_{j}=1 / k$, for $3 \leq j \leq m$, where $k \geq 1$. By assumption, for each such $k$, there exists a corresponding vector $\mathbf{y}^{k} \in F$ such that

$$
d_{1} x_{1} \overline{y_{1}^{k}}+d_{2} x_{2} \overline{y_{2}^{k}}+\frac{1}{k} \sum_{i=3}^{m} x_{j} \overline{y_{j}^{k}}=0 .
$$

Since $F$ is finite, there exists $\mathbf{y} \in F$ appearing infinitely many times in the collection $\left\{\mathbf{y}^{k}\right\}$. Letting $k$ go to infinity, it thus follows thus that

$$
d_{1} x_{1} \overline{y_{1}}+d_{2} x_{2} \overline{y_{2}}=0 .
$$

Since the set of solutions $\left(y_{1}, y_{2}\right) \in \mathbf{C}^{2}$ of (4.1) has the form $\left\{c\left(-d_{2} \overline{x_{2}}, d_{1} \overline{x_{1}}\right), c \in\right.$ $\mathbf{C}\}$, the ratio $\left|y_{2}\right| /\left|y_{1}\right|$ must be unbounded as we let $d_{1}$ and $d_{2}$ vary arbitrarily. This contradicts the fact that $F$ is finite.

Definition 4.6. If $\alpha_{1}, \ldots, \alpha_{m} \in \mathbf{T}^{n}$, we define

$$
\Omega\left(\alpha_{1}, \ldots, \alpha_{m}\right)=\left\{\beta \in \mathbf{T}^{n}, P(\beta)=0, \forall P \in \Pi_{\Lambda} \mid P\left(\alpha_{i}\right)=0, \forall i=1, \ldots, m\right\} .
$$

It is clear, from the previous definition, that $\left\{\alpha_{1}, \ldots, \alpha_{m}\right\} \subset \Omega\left(\alpha_{1}, \ldots, \alpha_{m}\right)$, but, in general, $\Omega\left(\alpha_{1}, \ldots, \alpha_{m}\right)$ might be strictly larger.

The following result gives us a simple way of checking that a set fails to have the extension property. Although we do not know if this property is equivalent to the failure of the extension property for general sets in dimensions higher than one, we will show that it is equivalent to it in dimension one. In particular, we will obtain a simple characterization of the sets with the extension property in that case. We will also show that all the known higher-dimensional examples of sets failing to have the extension property, as well as many other ones, can be obtained very easily from that result.

Theorem 4.7. Let $\Lambda$ be a finite subset of $\mathbf{Z}^{n}$. The following are equivalent: 
(a) There exist $\alpha_{1}, \ldots, \alpha_{m} \in \mathbf{T}^{n}$ such that $\Omega\left(\alpha_{1}, \ldots, \alpha_{m}\right)$ is a finite set strictly larger than $\left\{\alpha_{1}, \ldots, \alpha_{m}\right\}$.

(b) There exist $k$ linearly independent polynomials $P_{1}, \ldots, P_{k} \in \Pi_{\Lambda}$ such that the set $\bigcap_{i=1}^{k}\left\{\alpha \in \mathbf{T}^{n}, P_{i}(\alpha)=0\right\}$ is a finite set with cardinality greater than $|\Lambda|-k$.

Furthermore, if, in addition, $G(\Lambda-\Lambda)=\mathbf{Z}^{n}$, any one of these two conditions implies the failure of the extension property for $\Lambda$.

Proof. We will first prove the equivalence of (a) and (b). If (a) holds, we can assume, without loss of generality, that the linear functionals $L_{\alpha_{i}}, i=1, \ldots, m$, are linearly independent. Let $\mathcal{N}$ be the subspace of $\Pi_{\Lambda}$ consisting of the polynomials vanishing at all the $\alpha_{i}$ 's and choose $P_{1}, \ldots, P_{k}$ to be a basis for $\mathcal{N}$, where $k=|\Lambda|-m$. Then, clearly, $\bigcap_{i=1}^{k}\left\{\alpha \in \mathbf{T}^{n}, P_{i}(\alpha)=0\right\}=\Omega\left(\alpha_{1}, \ldots, \alpha_{m}\right)$ which is a set of cardinality greater than $m=|\Lambda|-k$. Conversely, if (b) holds, the set of linear functionals $\left\{L_{\alpha}\right\}_{\alpha \in A}$, where $A=\bigcap_{i=1}^{k}\left\{\beta \in \mathbf{T}^{n}, P_{i}(\beta)=0\right\}$ cannot be linearly independent. Let $\left\{\alpha_{1}, \ldots \alpha_{m}\right\}$ be a maximal subset of $A$ with the property that the collection $\left\{L_{\alpha_{i}}\right\}_{i=1}^{m}$ is linearly independent. Then, $\Omega\left(\alpha_{1}, \ldots, \alpha_{m}\right)=\bigcap_{i=1}^{k}\left\{\beta \in \mathbf{T}^{n}, P_{i}(\beta)=\right.$ $O\}$ which is a finite set strictly larger than $\left\{\alpha_{1}, \ldots, \alpha_{m}\right\}$. Hence, (a) holds. We will now prove that, under the additional assumption that $G(\Lambda-\Lambda)=\mathbf{Z}^{n}$, condition (a) implies the failure of the extension property for $\Lambda$. If some collection of points $\alpha_{1}, \ldots, \alpha_{m} \in \mathbf{T}^{n}$ satisfies the condition in (a), there is no loss of generality in assuming that the linear functionals $L_{\alpha_{i}}, i=1, \ldots, m$, are linearly independent. We can thus pick polynomials $U_{i} \in \Pi_{\Lambda}, i=1, \ldots, m$, satisfying $U_{i}\left(\alpha_{j}\right)=\delta_{i j}$, for $1 \leq$ $i, j \leq m$. Given any numbers $d_{1}, \ldots, d_{m}>0$, we define $\|P\|^{2}=\sum_{i=1}^{m} d_{i}\left|P\left(\alpha_{i}\right)\right|^{2}$ for $P \in \Pi_{\Lambda}$ and we denote by $E_{\beta}, \beta \in \Omega\left(\alpha_{1}, \ldots, \alpha_{m}\right)$, the associated evaluation polynomials and by $[.,$.$] the associated degenerate inner product. (Note that under$ our assumptions, $m<|\Lambda|$.) We notice that we can take $E_{\alpha_{i}}=U_{i} / d_{i}$. Indeed, if $P \in \Pi_{\Lambda}$, we have that

$$
\left[P, U_{i} / d_{i}\right]=\sum_{j=1}^{m} d_{j} P\left(\alpha_{j}\right) \overline{U_{i}\left(\alpha_{j}\right) / d_{i}}=P\left(\alpha_{i}\right), \quad i=1, \ldots, m .
$$

Since, by Lemma 3.8, the collection $\left\{E_{\alpha_{i}} /\left\|E_{\alpha_{i}}\right\|\right\}_{i=1}^{m}$ forms an orthonormal basis for $\Pi_{\Lambda}$ with respect to the inner product [.,.], it follows that, for any $\beta \in \Omega\left(\alpha_{1}, \ldots, \alpha_{m}\right)$, we can take for evaluation polynomial at $\beta$ the polynomial

$$
E_{\beta}=\sum_{i=1}^{m}\left[E_{\beta}, E_{\alpha_{i}}\right] E_{\alpha_{i}} /\left\|E_{\alpha_{i}}\right\|^{2}=\sum_{i=1}^{m}\left(1 / d_{i}\right) \overline{U_{i}(\beta)} U_{i} .
$$

Let $F=\left\{\left(U_{1}(\beta), \ldots, U_{m}(\beta)\right), \beta \in \Omega\left(\alpha_{1}, \ldots, \alpha_{m}\right)\right\} \subset \mathbf{C}^{m}$. Since $\Omega\left(\alpha_{1}, \ldots, \alpha_{m}\right)$ is assumed to be a finite set, so must be $F$. Now, let $\beta \in \Omega\left(\alpha_{1}, \ldots, \alpha_{m}\right) \backslash\left\{\alpha_{1}, \ldots, \alpha_{m}\right\}$. We claim that the vector $\left(U_{1}(\beta), \ldots, U_{m}(\beta)\right) \in F$ has, at least, two non-zero components. Indeed, if it weren't the case, it would follow from (4.2) that $E_{\beta}=c E_{\alpha_{j}}$, for some $c \in \mathbf{C}$ and $1 \leq j \leq m$, and thus that $P(\beta)=c P\left(\alpha_{j}\right)$, for all $P \in \Pi_{\Lambda}$. In particular, for the polynomial $\mathbf{z}^{\mathbf{k}}$, where $\mathbf{k} \in \Lambda$, we obtain that $\beta^{\mathbf{k}}=c \alpha_{j}^{\mathbf{k}}$ and, thus, that $|c|=1$. This implies that $|P(\beta)|^{2}=\left|P\left(\alpha_{j}\right)\right|^{2}$, for all $P \in \Pi_{\Lambda}$, contradicting the fact that $G(\Lambda-\Lambda)=\mathbf{Z}^{n}$ by Lemma 4.2. If $\Lambda$ has the extension property, Theorem 3.9 implies that, for any choice of numbers $d_{1}, \ldots, d_{m}$, there must be a corresponding element $\gamma \in \Omega\left(\alpha_{1}, \ldots, \alpha_{m}\right)$ such that $E_{\beta}(\gamma)=0$. In particular, we would then be able to find, for any such $d_{1}, \ldots, d_{m}$, a vector $\mathbf{y}=\left(y_{1}, \ldots, y_{m}\right) \in F$ 
such that $\sum_{i=1}^{m}\left(1 / d_{i}\right) U_{i}(\beta) \overline{y_{i}}=0$. Since $F$ is finite, this contradicts Lemma 4.5 and proves the claim.

To conclude this section, we will give examples of sets for which the failure of the extension property follows almost immediately from the previous result. In particular, the one-dimensional sets having the extension property are characterized.

Theorem 4.8. Any finite set $\Lambda \subset \mathbf{Z}$ having the extension property has the form

$$
\Lambda=\{k+j l, 0 \leq j \leq N\},
$$

for some $k, l, N \in \mathbf{Z}$ with $l, N \geq 0$.

Proof. Let us first assume that $\{0, N\} \subset \Lambda \subset\{i, 0 \leq i \leq N\}$, where $N$ is a non-negative integer, and that $G(\Lambda-\Lambda)=\mathbf{Z}$. If $\Lambda \neq\{i, 0 \leq i \leq N\}$, then the polynomial $z^{N}-1 \in \Pi_{\Lambda}$ has $N$ distinct roots on $\mathbf{T}$ while $|\Lambda|<N+1$, and thus $\Lambda$ fails to satisfy the extension property by Theorem 4.7 (using (b) with $k=1$ ). On the other hand if $\Lambda=\{i, 0 \leq i \leq N\}$, then $\Lambda$ is known to have the extension property (see $[\mathrm{Ru}])$. In the general case, the result can be obtained from the previous case by using Lemma 4.3 and Lemma 4.4, since the one-to-one homomorphisms of the group $\mathbf{Z}$ to itself are of the form $k \mapsto l k$, for some $l \in \mathbf{Z}, l \neq 0$.

We will now turn to the two-dimensional case and consider a class of examples of sets failing to satisfy the extension property. In particular, this class contains the sets considered by W. Rudin in $[\mathrm{Ru}]$ and the set $R(2,2)$ considered by A. Sakhnovich in $[\mathrm{Sa}]$.

Theorem 4.9. Let $\Lambda=R(p, q)$, with $p, q \geq 2$ or, more generally, let $\Lambda$ be any subset of $\mathbf{Z}^{2}$ containing the points $(0,0),(p, 0),(0, q),(p, q)$, where $p, q \geq 2$, and satisfying $|\Lambda| \leq(p+1)(q+1)$ as well as $G(\Lambda-\Lambda)=\mathbf{Z}^{2}$. Then, the extension property fails for $\Lambda$.

Proof. Notice first that the polynomial $1+z_{1}+z_{2}$ has exactly two roots on $\mathbf{T}^{2}$, $(\alpha, \bar{\alpha})$ and $(\bar{\alpha}, \alpha)$, where $\alpha=e^{2 \pi i / 3}$. Therefore, the polynomial $1+z_{1}^{p}+z_{2}^{q} \in \Pi_{\Lambda}$ has $2 p q$ roots on $\mathbf{T}^{2}$. If $p, q \geq 2$ and, either $p \geq 3$ or $q \geq 3$, we have that $|\Lambda| \leq(p+1)(q+1) \leq 2 p q$; thus, $\Lambda$ fails to satisfy the extension property, since the condition (b) of Theorem 4.7 is satisfied here with $k=1$. If $p=q=2$, the argument just given works also, except for $R(2,2)$ since, then, $|\Lambda|=9$ is greater than the number of zeros of $1+z_{1}^{2}+z_{2}^{2}$ on $\mathbf{T}^{2}$ which is 8 . Fortunately, condition (b) with $k=2$ is satisfied here by the polynomials $1-z_{1}^{2} z_{2}^{2}$ and $z_{1}^{2}-z_{2}^{2}$, since, as can be easily checked, the set of their common zeros on $\mathbf{T}^{2}$ has cardinality 8 , proving the claim.

Remark 4.10. Although, it is true that, in dimension greater than one, a trigonometric polynomial could have, in general, infinitely many zeros, one can actually show that if a polynomial in $\Pi_{\Lambda}$, where $\Lambda=R(2,2)$, only has finitely many zeros, then the number of its zeros is at most 8 . Hence, condition (b) of Theorem 4.7 with $k=1$ cannot be used to prove the failure of the extension property for $R(2,2)$. This raises the more general problem, unsolved as far as we know, as to how many zeros a polynomial in $\Pi_{\Lambda}$ can have if one knows, a priori, that it only has finitely many of them.

Finally, let us show how to use the previous results to give a simple proof of the failure of the extension property in 3-dimensional examples and, in particular, in the case of $\Lambda=R(1,1,1)$, a result first obtained by L. A. Sakhnovich ([Sa]). 
Proposition 4.11. The extension property fails for $\Lambda=R(p, q, r)$, for any integers $p, q, r \geq 1$.

Proof. The set of common zeros of the polynomials $\left(z_{1}-1\right)\left(z_{2} z_{3}+1\right),\left(z_{2}-1\right)\left(z_{1} z_{3}+\right.$ $1)$, and $\left(z_{3}-1\right)\left(z_{1} z_{2}+1\right)$ consists of the points

$$
\begin{gathered}
\alpha_{1}=(1,1,1), \alpha_{2}=(1,-1,-1), \alpha_{3}=(-1,1,-1), \\
\alpha_{4}=(-1,-1,1), \alpha_{5}=(i, i, i), \alpha_{6}=(-i,-i,-i)
\end{gathered}
$$

in $\mathbf{T}^{3}$. This implies that the set of common zeros of the polynomials $\left(z_{1}^{p}-1\right)\left(z_{2}^{q} z_{3}^{r}+\right.$ 1), $\left(z_{2}^{q}-1\right)\left(z_{1}^{p} z_{3}^{r}+1\right)$ and $\left(z_{3}^{r}-1\right)\left(z_{1}^{p} z_{2}^{q}+1\right)$ belonging to $\Pi_{\Lambda}$ has cardinality $6 p q r$. Since $|\Lambda|=(p+1)(q+1)(r+1)$, it follows, from using part (b) of Theorem 4.7 with $k=3$ and the fact that $G(\Lambda-\Lambda)=\mathbf{Z}^{3}$, that the extension property fails whenever

$$
6 p q r>(p+1)(q+1)(r+1)-3,
$$

an inequality which is satisfied if $p, q, r \geq 1$.

\section{Commuting Unitary matrices}

In this section, we will formulate a condition in terms of unitary matrices which is equivalent to the extension property and we will give some examples illustrating how this new criterion can be applied to show that a set does or does not have the extension property.

Definition 5.1. If $\mathbf{U}=\left(U_{1}, \ldots, U_{n}\right)$ is an $n$-tuple of unitary matrices $U_{i} \in \mathbf{M}_{r}$, for all $i=1, \ldots, n$, and $\mathbf{k}=\left(k_{1}, \ldots, k_{n}\right) \in \mathbf{Z}^{n}$, we will denote by $\mathbf{U}^{\mathbf{k}}$ the unitary matrix $U_{1}^{k_{1}} \ldots U_{n}^{k_{n}}$. Furthermore, $\mathbf{U}$ will be called a commuting $n$-tuple of unitary matrices if $U_{i} U_{j}=U_{j} U_{i}$, for all $i, j=1, \ldots, n$. If $\mathbf{x}, \mathbf{y} \in \mathbf{C}^{r}$, we will denote by $\mathbf{x} . \mathbf{y}$ the standard inner product in $\mathbf{C}^{r}$ of $\mathbf{x}$ and $\mathbf{y}$, and by $|\mathbf{x}|$, the standard euclidean norm of $\mathbf{x}$ in $\mathbf{C}^{r}$.

Theorem 5.2. Let $\Lambda$ be a finite subset of $\mathbf{Z}^{n}$. Then, $\Lambda$ satisfies the extension property if and only if, given any integer $r$, with $1 \leq r<|\Lambda|$, and any collection of vectors $\left\{\mathbf{x}_{\mathbf{k}}\right\}_{\mathbf{k} \in \Lambda}$ in $\mathbf{C}^{r}$ satisfying $\left|\mathbf{x}_{\mathbf{k}}\right|=1$, for all $\mathbf{k} \in \Lambda, \mathbf{x}_{\mathbf{k}} \cdot \mathbf{x}_{\mathbf{l}}=\mathbf{x}_{\mathbf{p}} \cdot \mathbf{x}_{\mathbf{q}}$ if $\mathbf{k}, \mathbf{l}, \mathbf{p}, \mathbf{q} \in \Lambda$ and $\mathbf{k}-\mathbf{l}=\mathbf{p}-\mathbf{q}$, and $\operatorname{span}\left\{\mathbf{x}_{\mathbf{k}}, \mathbf{k} \in \Lambda\right\}=\mathbf{C}^{r}$, there exists a commuting $n$-tuple of unitary matrices $\mathbf{U}=\left(U_{1}, \ldots, U_{n}\right)$, with $U_{i} \in \mathbf{M}_{r}$, for $i=1, \ldots, n$, and $\mathbf{x} \in \mathbf{C}^{r}$ such that $\mathbf{x}_{\mathbf{k}}=\mathbf{U}^{\mathbf{k}} \mathbf{x}$, for all $\mathbf{k} \in \Lambda$.

Proof. By Lemma 3.8, $\Lambda$ has the extension property if and only if, for every seminorm $\|$.$\| on \Pi_{\Lambda}$ associated with a collection p.d. on $\Lambda$, there exist $\alpha_{1}, \ldots \alpha_{r} \in \mathbf{T}^{n}$ and $d_{1}, \ldots, d_{r}>0$, where $r=|\Lambda|-\operatorname{dim}\left\{P \in \Pi_{\Lambda},\|P\|=0\right\}$, such that for all $P \in \Pi_{\Lambda}$,

$$
\|P\|^{2}=\sum_{i=1}^{r} d_{i}\left|P\left(\alpha_{i}\right)\right|^{2} .
$$

In fact, the same condition, but with the restriction that the collections considered be p.d. on $\Lambda$, but not s.p.d. on $\Lambda$, is also equivalent to the extension property. Indeed, assuming that this condition is satisfied, let us consider a collection which is s.p.d. on $\Lambda$ and let $\|$.$\| be the associated norm and let E_{\alpha}, \alpha \in \mathbf{T}^{n}$, be any associated evaluation polynomial. Then,

$$
\|P\|_{1}^{2}=\|P\|^{2}-|P(\alpha)|^{2} /\left\|E_{\alpha}\right\|^{2}
$$


defines a semi-norm associated with a p.d. collection which is not s.p.d. on $\Lambda$, since the set $\left\{P \in \Pi_{\Lambda},\|P\|_{1}=0\right\}$ is the one-dimensional subspace generated by $E_{\alpha}$. By assumption, we can write

$$
\|P\|_{1}^{2}=\sum_{i=1}^{|\Lambda|-1} d_{i}\left|P\left(\alpha_{i}\right)\right|^{2},
$$

where the $\alpha_{i}$ 's are in $\mathbf{T}^{n}$ and the $d_{i}$ 's are positive, from which a representation as in (5.1) with $r=|\Lambda|$ follows for the original norm. Let us thus consider a collection $\left\{c_{\mathbf{k}}\right\}_{\mathbf{k} \in \Lambda-\Lambda}$ which is p.d., but not s.p.d. on $\Lambda$, and let $\|$.$\| be the associated semi-$ norm. Let $L_{1}, \ldots, L_{r}$ be $r$ linearly independent linear functionals on $\Pi_{\Lambda}$ such that

$$
\|P\|^{2}=\sum_{i=1}^{r}\left|L_{i}(P)\right|^{2} .
$$

If $L_{i}(P)=\sum_{\mathbf{k} \in \Lambda} u_{\mathbf{k}}^{i} a_{\mathbf{k}}$, for $P(\mathbf{z})=\sum_{\mathbf{k} \in \Lambda} a_{\mathbf{k}} \mathbf{z}^{\mathbf{k}} \in \Pi_{\Lambda}$, we define a collection of vectors in $\mathbf{C}^{r},\left\{\mathbf{u}_{\mathbf{k}}\right\}_{\mathbf{k} \in \Lambda}$, by $\left(\mathbf{u}_{\mathbf{k}}\right)_{i}=u_{\mathbf{k}}^{i}, i=1, \ldots, r$. There is no loss of generality in assuming that $\left\|\mathbf{z}^{\mathbf{k}}\right\|=1$, for all $\mathbf{k} \in \Lambda$. Since

$$
\|P\|^{2}=\sum_{\mathbf{k}, \mathbf{l} \in \Lambda}\left\{\sum_{i=1}^{r} u_{\mathbf{k}}^{i} \overline{u_{1}^{i}}\right\} a_{\mathbf{k}} \overline{a_{\mathbf{l}}}
$$

it follows that

$$
\left|\mathbf{u}_{\mathbf{k}}\right|^{2}=1, \quad \mathbf{k} \in \Lambda
$$

and that

$$
\mathbf{u}_{\mathbf{k}} \cdot \mathbf{u}_{\mathbf{l}}=\mathbf{u}_{\mathbf{p}} \cdot \mathbf{u}_{\mathbf{q}}, \quad \forall \mathbf{k}, \mathbf{l}, \mathbf{p}, \mathbf{q} \in \Lambda, \text { with } \mathbf{k}-\mathbf{l}=\mathbf{p}-\mathbf{q} .
$$

Furthermore, the span of $\left\{\mathbf{u}_{\mathbf{k}}\right\}_{\mathbf{k} \in \Lambda}$ is all of $\mathbf{C}^{r}$. Indeed, if for some $\mathbf{x}=\left(x_{1}, \ldots, x_{r}\right) \in$ $\mathbf{C}^{r}$, we had $\sum_{i=1}^{r} x_{i} u_{\mathbf{k}}^{i}=0$, for all $\mathbf{k} \in \Lambda$, this would imply that $\sum_{i=1}^{r} x_{i} L_{i}(P)=0$, for all $P \in \Pi_{\Lambda}$, and, thus, that all the $x_{i}$ 's are zero, since the linear functionals $L_{i}, i=1, \ldots, r$, are linearly independent. Hence, any semi-norm associated with a collection p.d., but not s.p.d., on $\Lambda$, can be represented, if $\left\|\mathbf{z}^{\mathbf{k}}\right\|=1$, for all monomials $\mathbf{z}^{\mathbf{k}}, \mathbf{k} \in \Lambda$, in the form

$$
\|P\|^{2}=\sum_{\mathbf{k}, \mathbf{l} \in \Lambda} \mathbf{u}_{\mathbf{k}} \cdot \mathbf{u}_{\mathbf{l}} a_{\mathbf{k}} \overline{a_{\mathbf{l}}}=\left|\sum_{\mathbf{k} \in \Lambda} a_{\mathbf{k}} \mathbf{u}_{\mathbf{k}}\right|^{2},
$$

for $P(\mathbf{z})=\sum_{\mathbf{k} \in \Lambda} a_{\mathbf{k}} \mathbf{z}^{\mathbf{k}} \in \Pi_{\Lambda}$, where the vectors $\mathbf{u}_{\mathbf{k}}$ belong to $\mathbf{C}^{r}$, for some $1 \leq r<$ $|\Lambda|$, and satisfy (5.2) and (5.3). Assuming now that $\Lambda$ has the extension property, there will exist, by Lemma 3.8, $\alpha_{1}, \ldots, \alpha_{r} \in \mathbf{T}^{n}$ and $d_{1}, \ldots, d_{r}>0$ such that the linear functionals $L_{\alpha_{i}}, i=1, \ldots, r$, are linearly independent and for which the identity

$$
\|P\|^{2}=\sum_{i=1}^{r} d_{i}\left|P\left(\alpha_{i}\right)\right|^{2}
$$

is satisfied for all $P \in \Pi_{\Lambda}$. Consider the collection $\left\{\mathbf{v}_{\mathbf{k}}\right\}_{\mathbf{k} \in \Lambda}$ of vectors in $\mathbf{C}^{r}$, defined by $\left(\mathbf{v}_{\mathbf{k}}\right)_{i}=\sqrt{d_{i}} \alpha_{i}^{\mathbf{k}}$, for $1 \leq i \leq r$. It follows from (5.4) that

$$
\|P\|^{2}=\sum_{\mathbf{k}, \mathbf{l} \in \Lambda} \mathbf{v}_{\mathbf{k}} \cdot \mathbf{v}_{\mathbf{l}} a_{\mathbf{k}} \overline{a_{\mathbf{l}}},
$$


for all $P(\mathbf{z})=\sum_{\mathbf{k} \in \Lambda} a_{\mathbf{k}} \mathbf{z}^{\mathbf{k}} \in \Pi_{\Lambda}$, and, thus, comparing (5.3) and (5.5), that

$$
\mathbf{u}_{\mathbf{k}} \cdot \mathbf{u}_{\mathbf{l}}=\mathbf{v}_{\mathbf{k}} \cdot \mathbf{v}_{\mathbf{l}}, \quad \mathbf{k}, \mathbf{l} \in \Lambda \text {. }
$$

Consider now the linear map $A: \mathbf{C}^{r} \rightarrow \mathbf{C}^{r}$ defined by

$$
A\left(\sum_{\mathbf{k} \in \Lambda} x_{\mathbf{k}} \mathbf{u}_{\mathbf{k}}\right)=\sum_{\mathbf{k} \in \Lambda} x_{\mathbf{k}} \mathbf{v}_{\mathbf{k}},
$$

where $x_{\mathbf{k}} \in \mathbf{C}$, for all $\mathbf{k} \in \Lambda$. Because of (5.6), we have that

$$
\left|\sum_{\mathbf{k} \in \Lambda} x_{\mathbf{k}} \mathbf{v}_{\mathbf{k}}\right|^{2}=\sum_{\mathbf{k}, \mathbf{l} \in \Lambda} \mathbf{v}_{\mathbf{k}} \cdot \mathbf{v}_{\mathbf{l}} x_{\mathbf{k}} \overline{x_{\mathbf{l}}}=\sum_{\mathbf{k}, \mathbf{l} \in \Lambda} \mathbf{u}_{\mathbf{k}} \cdot \mathbf{u}_{\mathbf{l}} x_{\mathbf{k}} \overline{x_{\mathbf{l}}}=\left|\sum_{\mathbf{k} \in \Lambda} x_{\mathbf{k}} \mathbf{u}_{\mathbf{k}}\right|^{2}
$$

and, thus, $A$ is a well-defined unitary map from $\mathbf{C}^{r}$ to $\mathbf{C}^{r}$. Let us define $\mathbf{v} \in \mathbf{C}^{r}$ by $\mathbf{v}_{i}=\sqrt{d_{i}}$, for $i=1, \ldots, r$ and $n$ diagonal unitary matrices $V_{j} \in \mathbf{M}_{r}, j=1, \ldots, n$, by $V_{j}=\operatorname{diag}\left(\left(\alpha_{1}\right)_{j}, \ldots,\left(\alpha_{r}\right)_{j}\right)$ where $\left(\alpha_{k}\right)_{j}$ denotes the $j$-th component of $\alpha_{k} \in \mathbf{T}^{n}$. Clearly, $V_{i} V_{j}=V_{j} V_{i}$, for all $i, j=1, \ldots, n$ and $\mathbf{v}_{\mathbf{k}}=V_{1}^{k_{1}} \ldots V_{n}^{k_{n}} \mathbf{v}$, for all $\mathbf{k} \in \Lambda$. Hence, letting $\mathbf{V}$ be the commuting $n$-tuple of unitary matrices $\left(V_{1}, \ldots, V_{n}\right)$, we have that $\mathbf{v}_{\mathbf{k}}=\mathbf{V}^{\mathbf{k}} \mathbf{v}=\mathbf{V}^{\mathbf{k}} A \mathbf{u}$, where $\mathbf{u}=A^{*} \mathbf{v}$, for each $\mathbf{k} \in \Lambda$. Hence, letting $\mathbf{U}$ be the commuting $n$-tuple of unitary matrices $\left(U_{1}, \ldots, U_{n}\right)$, where $U_{i}=A^{*} V_{i} A$, for $i=1, \ldots, n$, it follows, using the identity $A^{*} A=I$, that if $\mathbf{k} \in \Lambda$, we have

$$
\mathbf{u}_{\mathbf{k}}=A^{*} \mathbf{V}^{\mathbf{k}} A \mathbf{u}=\mathbf{U}^{\mathbf{k}} \mathbf{u}
$$

as was claimed. Conversely, assuming that for any collection of vectors $\left\{\mathbf{u}_{\mathbf{k}}\right\}_{\mathbf{k} \in \Lambda}$ in $\mathbf{C}^{r}, 1 \leq r<|\Lambda|$, spanning all of $\mathbf{C}^{r}$ and satisfying (5.2) and (5.3), there exists a commuting $n$-tuple $\mathbf{U}$ of unitary matrices in $\mathbf{M}_{r}$ and $\mathbf{u} \in \mathbf{C}^{r}$ such that $\mathbf{u}_{\mathbf{k}}=\mathbf{U}^{\mathbf{k}} \mathbf{u}$, for all $\mathbf{k} \in \Lambda$, let us consider a collection $\left\{c_{\mathbf{k}}\right\}_{\mathbf{k} \in \Lambda-\Lambda}$ p.d., but not s.p.d., on $\Lambda$. By the above considerations, we can find a collection of vectors $\left\{\mathbf{u}_{\mathbf{k}}\right\}_{\mathbf{k} \in \Lambda}$ in $\mathbf{C}^{r}$, where $r=|\Lambda|-\operatorname{dim}\left\{P \in \Pi_{\Lambda},\|P\|=0\right\}$, whose span is all of $\mathbf{C}^{r}$ and such that, for all $P \in \Pi_{\Lambda}$, with $P(\mathbf{z})=\sum_{\mathbf{k} \in \Lambda} a_{\mathbf{k}} \mathbf{z}^{\mathbf{k}}$,

$$
\|P\|^{2}=\sum_{\mathbf{k}, \mathbf{l} \in \Lambda} \mathbf{u}_{\mathbf{k}} \cdot \mathbf{u}_{\mathbf{l}} a_{\mathbf{k}} \overline{a_{\mathbf{l}}}
$$

By assumption, there is thus an $n$-tuple of unitary matrices $\mathbf{U}=\left(U_{1}, \ldots, U_{n}\right)$ in $\mathbf{M}_{r}$ and $\mathbf{u} \in \mathbf{C}^{r}$ such that $\mathbf{u}_{\mathbf{k}}=\mathbf{U}^{\mathbf{k}} \mathbf{u}$, for all $\mathbf{k} \in \Lambda$. Since the matrices $U_{i}$ 's commute with each other, they are simultaneously diagonalizable by a unitary similarity (see [HJ, p. 103]). There exists thus a unitary matrix $A \in \mathbf{M}_{r}$ such that $U_{i}=A^{*} V_{i} A$, for all $i=1, \ldots, n$, where $V_{i}$ is a diagonal matrix of the form $\operatorname{diag}\left(\left(\alpha_{1}\right)_{i}, \ldots,\left(\alpha_{r}\right)_{i}\right)$, and $\left(\alpha_{1}\right)_{i}, \ldots,\left(\alpha_{r}\right)_{i} \in \mathbf{T}$. Let us define $\alpha_{j} \in \mathbf{T}^{n}$, for $j=1, \ldots, r$, by $\alpha_{j}=$ $\left(\left(\alpha_{j}\right)_{1}, \ldots,\left(\alpha_{j}\right)_{n}\right)$ and the $n$-tuple $\mathbf{V}$ of unitary matrices by $\left(V_{1}, \ldots, V_{n}\right)$. If $P(\mathbf{z})=$ $\sum_{\mathbf{k} \in \Lambda} a_{\mathbf{k}} \mathbf{z}^{\mathbf{k}} \in \Pi_{\Lambda}$, we obtain, letting $\mathbf{v}=\left(v_{1}, \ldots, v_{r}\right)=A \mathbf{u}$, that

$$
\begin{aligned}
\|P\|^{2} & =\sum_{\mathbf{k}, \mathbf{l} \in \Lambda}\left(\mathbf{U}^{\mathbf{k}} \mathbf{u}\right) \cdot\left(\mathbf{U}^{\mathbf{l}} \mathbf{u}\right) a_{\mathbf{k}} \overline{a_{\mathbf{l}}}=\sum_{\mathbf{k}, \mathbf{l} \in \Lambda}\left(A^{*} \mathbf{V}^{\mathbf{k}} A \mathbf{u}\right) \cdot\left(A^{*} \mathbf{V}^{\mathbf{l}} A \mathbf{u}\right) a_{\mathbf{k}} \overline{a_{\mathbf{l}}} \\
& =\sum_{\mathbf{k}, \mathbf{l} \in \Lambda}\left(\mathbf{V}^{\mathbf{k}-\mathbf{l}} \mathbf{v}\right) \cdot \mathbf{v} a_{\mathbf{k}} \overline{a_{\mathbf{l}}}=\sum_{\mathbf{k}, \mathbf{l} \in \Lambda}\left\{\sum_{i=1}^{r}\left(\alpha_{i}\right)^{\mathbf{k}-\mathbf{l}}\left|\mathbf{v}_{i}\right|^{2}\right\} a_{\mathbf{k}} \overline{a_{\mathbf{l}}}=\sum_{i=1}^{r}\left|v_{i}\right|^{2}\left|P\left(\alpha_{i}\right)\right|^{2} .
\end{aligned}
$$

To conclude the proof, it remains to notice that $\left|v_{i}\right| \neq 0$ and that the linear functional $L_{\alpha_{i}}, i=1, \ldots, r$, are linearly independent, since the dimension of $\left\{P \in \Pi_{\Lambda},\|P\|=0\right\}$ would otherwise be greater than $|\Lambda|-r$. 
To illustrate the previous theorem, we will give a simple two-dimensional example of a set failing the extension property.

Example 5.3. The set $\Lambda=\{(0,0),(1,0),(2,0),(0,1),(1,1),(0,2)\} \subset \mathbf{Z}^{2}$ fails to satisfy the extension property. Indeed, let $\left\{\mathbf{u}_{i}, i=1, \ldots, 5\right\}$ be any orthonormal basis of $\mathbf{C}^{5}$ (with respect to the standard inner product) and define $\mathbf{x}_{(0,0)}=\mathbf{u}_{1}$, $\mathbf{x}_{(1,0)}=\mathbf{u}_{2}, \mathbf{x}_{(2,0)}=\mathbf{u}_{3}, \mathbf{x}_{(0,1)}=\mathbf{u}_{4}, \mathbf{x}_{(1,1)}=\mathbf{u}_{5}$, and $\mathbf{x}_{(0,2)}=\mathbf{u}_{1}$. It is easily checked that $\mathbf{x}_{\mathbf{k}} \cdot \mathbf{x}_{\mathbf{l}}=\mathbf{x}_{\mathbf{p}} \cdot \mathbf{x}_{\mathbf{q}}$ if $\mathbf{k}, \mathbf{l}, \mathbf{p}, \mathbf{q} \in \Lambda$ and $\mathbf{k}-\mathbf{l}=\mathbf{p}-\mathbf{q}$. Suppose now that there is a commuting pair of unitary matrices $\mathbf{U}=\left(U_{1}, U_{2}\right)$ in $\mathbf{M}_{5}$ such that $\mathbf{x}_{\mathbf{k}}=\mathbf{U}^{\mathbf{k}} \mathbf{x}_{(0,0)}$, for all $\mathbf{k} \in \Lambda$. Let $\mathbf{x}_{\mathbf{k}}=\mathbf{U}^{\mathbf{k}} \mathbf{x}_{(0,0)}$, for all $\mathbf{k} \in \mathbf{Z}^{2}$. Then we have that $\mathbf{x}_{(1,0)} \cdot \mathbf{x}_{(1,2)}=\mathbf{x}_{(0,0)} \cdot \mathbf{x}_{(0,2)}=\left|\mathbf{u}_{1}\right|^{2}=1$. Since the $\mathbf{x}_{\mathbf{k}}$ 's are all unit vectors, it follows that $\mathbf{x}_{(1,2)}=\mathbf{x}_{(1,0)}=\mathbf{u}_{2}$. Similarly, $\mathbf{x}_{(2,2)}=\mathbf{x}_{(0,2)}=\mathbf{u}_{3}$. Consider now the vector $\mathbf{x}_{(2,1)}$. We have that $\mathbf{x}_{(2,1)} \cdot \mathbf{x}_{(0,0)}=\mathbf{x}_{(2,2)} \cdot \mathbf{x}_{(0,1)}=\mathbf{u}_{3} \cdot \mathbf{u}_{4}=0$, $\mathbf{x}_{(2,1)} \cdot \mathbf{x}_{(1,0)}=\mathbf{x}_{(1,1)} \cdot \mathbf{x}_{(0,0)}=\mathbf{u}_{5} \cdot \mathbf{u}_{1}=0, \mathbf{x}_{(2,1)} \cdot \mathbf{x}_{(2,0)}=\mathbf{x}_{(0,1)} \cdot \mathbf{x}_{(0,0)}=\mathbf{u}_{4} \cdot \mathbf{u}_{1}=0$, $\mathbf{x}_{(2,1)} \cdot \mathbf{x}_{(1,1)}=\mathbf{x}_{(1,0)} \cdot \mathbf{x}_{(0,0)}=\mathbf{u}_{2} \cdot \mathbf{u}_{1}=0$. Hence, $\mathbf{x}_{(2,1)}$ must be orthogonal to each vector $\mathbf{u}_{i}, i=1, \ldots, 5$, contradicting the fact that it is a unit vector.

Definition 5.4. If a collection $\left\{c_{\mathbf{k}}\right\}_{\mathbf{k} \in \Lambda-\Lambda}$ is p.d. on $\Lambda$, we defined the rank of $\left\{c_{\mathbf{k}}\right\}_{\mathbf{k} \in \Lambda-\Lambda}$ to be the number $|\Lambda|-\operatorname{dim}\left\{P \in \Pi_{\Lambda},\|P\|=0\right\}$, where $\|$.$\| denotes the$ semi-norm associated with $\left\{c_{\mathbf{k}}\right\}_{\mathbf{k} \in \Lambda-\Lambda}$.

A consequence of Theorem 5.2 is the following result which applies to sets $\Lambda$ which are "path-connected" in the sense described below.

Corollary 5.5. Given a finite set $\Lambda \subset \mathbf{Z}^{n}$, let $\tilde{\Lambda}=\left\{\mathbf{k}+\sum_{i=1}^{n} \epsilon_{i} \mathbf{e}_{i}, \mathbf{k} \in \Lambda, \epsilon_{i}=\right.$ $0,1\}$, where $\mathbf{e}_{i}$ denotes the element of $\mathbf{Z}^{n}$ whose components are all zero, except for the $i$-th which is 1. Suppose, furthermore, that $\Lambda$ has the property that, for any distinct $\mathbf{k}, \mathbf{l} \in \Lambda$, there exists a finite sequence $\mathbf{p}_{1}, \ldots, \mathbf{p}_{m} \in \Lambda$ satisfying $\mathbf{p}_{1}=\mathbf{k}$, $\mathbf{p}_{m}=\mathbf{l}$, and $\mathbf{p}_{j+1}=\mathbf{p}_{j}+\eta_{j} \mathbf{e}_{i(j)}$, for all $j=1, \ldots, m-1$, where $\eta_{j}= \pm 1$ and $i(j) \in\{1, \ldots, n\}$. If, for any collection $\left\{c_{\mathbf{k}}\right\}_{\mathbf{k} \in \Lambda-\Lambda}$ p.d. on $\Lambda$ and of rank $r$, $1 \leq r<|\Lambda|$, there exists a collection $\left\{\tilde{c}_{\mathbf{k}}\right\}_{\mathbf{k} \in \tilde{\Lambda}-\tilde{\Lambda}}$ p.d. on $\tilde{\Lambda}$ and of rank $r$ such that $\tilde{c}_{\mathbf{k}}=c_{\mathbf{k}}$, for all $\mathbf{k} \in \Lambda-\Lambda$, then $\Lambda$ has the extension property.

Proof. As in the proof of Theorem 5.2, if $\|$.$\| denotes the semi-norm associated with$ a collection $\left\{c_{\mathbf{k}}\right\}_{\mathbf{k} \in \Lambda-\Lambda}$ p.d. on $\Lambda$ satisfying, without loss of generality $\left\|\mathbf{z}^{\mathbf{k}}\right\|=1$, for all $\mathbf{k} \in \Lambda$, there exists, by assumption, a collection $\left\{\mathbf{u}_{\mathbf{k}}\right\}_{\mathbf{k} \in \tilde{\Lambda}}$ in $\mathbf{C}^{r}$ whose span is all of $\mathbf{C}^{r}$ and which satisfies $\left|\mathbf{u}_{\mathbf{k}}\right|=1$, for all $\mathbf{k} \in \tilde{\Lambda}$ and $\mathbf{u}_{\mathbf{k}} \cdot \mathbf{u}_{\mathbf{l}}=\mathbf{u}_{\mathbf{p}} \cdot \mathbf{u}_{\mathbf{q}}$, for all $\mathbf{k}, \mathbf{l}, \mathbf{p}, \mathbf{q} \in \tilde{\Lambda}$ such that $\mathbf{k}-\mathbf{l}=\mathbf{p}-\mathbf{q}$, for which

$$
\|P\|^{2}=\left|\sum_{\mathbf{k} \in \Lambda} a_{\mathbf{k}} \mathbf{u}_{\mathbf{k}}\right|^{2},
$$

for all $P(\mathbf{z})=\sum_{\mathbf{k} \in \Lambda} a_{\mathbf{k}} \mathbf{z}^{\mathbf{k}} \in \Pi_{\Lambda}$. For each $i=1, \ldots, n$, we let $U_{i}: \mathbf{C}^{r} \rightarrow \mathbf{C}^{r}$ be the mapping defined by $U_{i}\left(\sum_{\mathbf{k} \in \Lambda} a_{\mathbf{k}} \mathbf{u}_{\mathbf{k}}\right)=\sum_{\mathbf{k} \in \Lambda} a_{\mathbf{k}} \mathbf{u}_{\mathbf{k}+\mathbf{e}_{i}}$. Each $U_{i}$ is unitary since $\mathbf{u}_{\mathbf{k}+\mathbf{e}_{i}} \cdot \mathbf{u}_{\mathbf{l}+\mathbf{e}_{i}}=\mathbf{u}_{\mathbf{k}} \cdot \mathbf{u}_{\mathbf{l}}$, for all $\mathbf{k}, \mathbf{l} \in \Lambda$. Furthermore, we claim that $U_{i} U_{j}=U_{j} U_{i}$ if $i \neq j$. Indeed, note that for all $\mathbf{k}, \mathbf{l} \in \Lambda$, we have

$$
U_{j} U_{i} \mathbf{u}_{\mathbf{k}} \cdot \mathbf{u}_{\mathbf{l}+\mathbf{e}_{j}}=U_{j} \mathbf{u}_{\mathbf{k}+\mathbf{e}_{i}} \cdot \mathbf{u}_{\mathbf{l}+\mathbf{e}_{j}}=\mathbf{u}_{\mathbf{k}+\mathbf{e}_{i}} \cdot U_{j}^{*} \mathbf{u}_{\mathbf{l}+\mathbf{e}_{j}}=\mathbf{u}_{\mathbf{k}+\mathbf{e}_{i}} \cdot \mathbf{u}_{\mathbf{l}}=\mathbf{u}_{\mathbf{k}+\mathbf{e}_{i}+\mathbf{e}_{j}} \cdot \mathbf{u}_{\mathbf{l}+\mathbf{e}_{j}},
$$

and, thus, since the span of the collection $\left\{\mathbf{u}_{\mathbf{l}+\mathbf{e}_{j}}\right\}_{\mathbf{l} \in \Lambda}$ is all of $\mathbf{C}^{r}$, it follows that

$$
U_{j} U_{i} \mathbf{u}_{\mathbf{k}}=\mathbf{u}_{\mathbf{k}+\mathbf{e}_{i}+\mathbf{e}_{j}}=U_{i} U_{j} \mathbf{u}_{\mathbf{k}}, \quad \mathbf{k} \in \Lambda,
$$


from which the desired commutativity follows immediately. Hence, $\mathbf{U}=\left(U_{1}, \ldots, U_{n}\right)$ defines a commuting $n$-tuple of unitary matrices. To conclude the proof, it remains to show that, for any $\mathbf{k}, \mathbf{l} \in \Lambda$, we have $\mathbf{u}_{\mathbf{l}}=\mathbf{U}^{\mathbf{l}-\mathbf{k}} \mathbf{u}_{\mathbf{k}}$, since, in this case, the desired conclusion will follow from Theorem 5.2. If $\mathbf{k}=\mathbf{l}$, this is clear, and if $\mathbf{k} \neq \mathbf{l}$, let $\mathbf{p}_{1}, \ldots, \mathbf{p}_{m} \in \Lambda$ be the corresponding finite sequence in the assumptions. Using the fact that, for all $j=1, \ldots, m-1$, we have that

$$
\mathbf{u}_{\mathbf{p}_{j+1}}=\mathbf{u}_{\mathbf{p}_{j}+\eta_{j} \mathbf{e}_{i(j)}}=U_{i(j)}^{\eta_{j}} \mathbf{u}_{\mathbf{p}_{j}}=\mathbf{U}^{\eta_{j} \mathbf{e}_{i(j)}} \mathbf{u}_{\mathbf{p}_{j}},
$$

we compute

$$
\begin{aligned}
\mathbf{U}^{\mathbf{l}-\mathbf{k}} \mathbf{u}_{\mathbf{k}} & =\mathbf{U}^{\mathbf{l}-\mathbf{k}} \mathbf{u}_{\mathbf{p}_{1}}=\mathbf{U}^{\mathbf{l}-\mathbf{k}} \mathbf{U}^{\left(-\eta_{1} \mathbf{e}_{i(1)}\right)} \mathbf{u}_{\mathbf{p}_{2}} \\
& =\mathbf{U}^{\left(\mathbf{l}-\mathbf{k}-\eta_{1} \mathbf{e}_{i(1)}\right)} \mathbf{u}_{\mathbf{p}_{2}}=\cdots=\mathbf{U}^{\left(\mathbf{l}-\mathbf{k}-\sum_{j=1}^{m-1} \eta_{j} \mathbf{e}_{i(j)}\right)} \mathbf{u}_{\mathbf{p}_{m}}=\mathbf{u}_{\mathbf{l}},
\end{aligned}
$$

since $\sum_{j=1}^{m-1} \eta_{j} \mathbf{e}_{i(j)}=\mathbf{l}-\mathbf{k}$, which concludes the proof.

Remark 5.6. The "path-connectednesss" property that $\Lambda$ must satisfy is really needed in the proof of the previous corollary. For example, in one dimension, if we take $\Lambda=\{0,1,6,7\}$, then $\tilde{\Lambda}=\{0,1,2,6,7,8\}$, and if we let $\mathbf{e}_{1}=(1,0,0)$, $\mathbf{e}_{2}=(0,1,0)$, and $\mathbf{e}_{3}=(0,0,1)$ be the standard orthonormal basis for $\mathbf{C}^{3}$ and define $\mathbf{u}_{0}=\mathbf{e}_{1}, \mathbf{u}_{1}=\mathbf{e}_{2}, \mathbf{u}_{2}=\mathbf{e}_{3}, \mathbf{u}_{6}=\mathbf{e}_{2}, \mathbf{u}_{7}=\mathbf{e}_{3}, \mathbf{u}_{8}=\mathbf{e}_{1}$, it is easily checked that $\mathbf{u}_{k} \cdot \mathbf{u}_{l}=\mathbf{u}_{p} \cdot \mathbf{u}_{q}$, for all $k, l, p, q \in \tilde{\Lambda}$ with $k-l=p-q$ and thus, the unitary operator $U$ appearing in the proof of Corollary 5.5 can be defined by $U\left(\mathbf{e}_{1}\right)=\mathbf{e}_{2}$, $U\left(\mathbf{e}_{2}\right)=\mathbf{e}_{3}, U\left(\mathbf{e}_{3}\right)=\mathbf{e}_{1}$. However, we have $U^{6}\left(\mathbf{u}_{0}\right)=\mathbf{u}_{0} \neq \mathbf{u}_{6}$.

We will now use the previous result to give an elementary proof of the well-known fact that the one-dimensional set $\{0,1, \ldots, N\}$ has the extension property.

Lemma 5.7. Let $\mathbf{x}_{0}, \ldots, \mathbf{x}_{k}, \mathbf{x}_{k+1}$ be $k+2$ vectors in $\mathbf{C}^{m}$ satisfying $\left|\mathbf{x}_{i}\right|=1$, for all $i=0, \ldots, k+1$, and $\mathbf{x}_{i} \cdot \mathbf{x}_{j}=\mathbf{x}_{p} \cdot \mathbf{x}_{q}$, for all $i, j, p, q \in\{0, \ldots, k+1\}$ with $i-j=p-q$. Then, if $\mathbf{x}_{k}$ belongs to the span of $\mathbf{x}_{0}, \ldots, \mathbf{x}_{k-1}$, so does $\mathbf{x}_{k+1}$.

Proof. By assumption, $\mathbf{x}_{k}=\sum_{i=0}^{k-1} a_{i} \mathbf{x}_{i}$, where $a_{i} \in \mathbf{C}$, for all $i=0, \ldots, k-1$. Let $\mathbf{u}=\mathbf{x}_{k+1}-\sum_{i=0}^{k-1} a_{i} \mathbf{x}_{i+1}$. Then, if $1 \leq l \leq k$, we have

$$
\begin{aligned}
\mathbf{u} \cdot \mathbf{x}_{l} & =\mathbf{x}_{k+1} \cdot \mathbf{x}_{l}-\sum_{i=0}^{k-1} a_{i} \mathbf{x}_{i+1} \cdot \mathbf{x}_{l}=\mathbf{x}_{k} \cdot \mathbf{x}_{l-1}-\sum_{i=0}^{k-1} a_{i} \mathbf{x}_{i} \cdot \mathbf{x}_{l-1} \\
& =\left(\mathbf{x}_{k}-\sum_{i=0}^{k-1} a_{i} \mathbf{x}_{i}\right) \cdot \mathbf{x}_{l-1}=0 .
\end{aligned}
$$

In particular, we obtain that $\mathbf{x}_{k+1}=\sum_{i=0}^{k-1} a_{i} \mathbf{x}_{i+1}+\mathbf{u}$, where $\mathbf{u} \cdot\left(\sum_{i=0}^{k-1} a_{i} \mathbf{x}_{i+1}\right)=$ 0 . Hence,

$$
1=\left|\mathbf{x}_{k+1}\right|^{2}=\left|\sum_{i=0}^{k-1} a_{i} \mathbf{x}_{i+1}\right|^{2}+|\mathbf{u}|^{2}=\left|\sum_{i=0}^{k-1} a_{i} \mathbf{x}_{i}\right|^{2}+|\mathbf{u}|^{2}=\left|\mathbf{x}_{k}\right|^{2}+|\mathbf{u}|^{2}=1+|\mathbf{u}|^{2},
$$

proving that $\mathbf{u}=0$ and, thus, that $\mathbf{x}_{k+1}=\sum_{i=0}^{k-1} a_{i} \mathbf{x}_{i+1}$ belongs to the span of the vectors $\mathbf{x}_{0}, \ldots, \mathbf{x}_{k-1}$.

Theorem 5.8 (See $[\mathrm{Ru}]$ ). The set $\{0,1, \ldots, N\}$ satisfies the extension property. 
Proof. By Corollary 5.5, we need to show that if $\mathbf{x}_{0}, \ldots, \mathbf{x}_{N}$ are $N+1$ unit vectors in $\mathbf{C}^{r}$, where $1 \leq r \leq N$, whose span is all of $\mathbf{C}^{r}$ and which satisfy $\mathbf{x}_{i} \cdot \mathbf{x}_{j}=\mathbf{x}_{p} \cdot \mathbf{x}_{q}$, for all $i, j, p, q \in\{0, \ldots, N\}$ with $i-j=p-q$, then, there exists a unit vector $\mathbf{x}_{N+1} \in \mathbf{C}^{r}$ such that $\mathbf{x}_{N+1} \cdot \mathbf{x}_{j}=\mathbf{x}_{N+1-p} \cdot \mathbf{x}_{j-p}$, for all $j \in\{1, \ldots, N\}$ and all $p \in\{1, \ldots, j\}$. It follows immediately from the previous lemma that the span of $\mathbf{x}_{0}, \ldots, \mathbf{x}_{r-1}$ is all of $\mathbf{C}^{r}$. Thus, if $\mathbf{x}_{N}=\sum_{i=0}^{r-1} a_{i} \mathbf{x}_{i}$, we let $\mathbf{x}_{N+1}=\sum_{i=0}^{r-1} a_{i} \mathbf{x}_{i+1}$, and, if $j, p$ are as above, we compute

$$
\mathbf{x}_{N+1} \cdot \mathbf{x}_{j}=\left(\sum_{i=0}^{r-1} a_{i} \mathbf{x}_{i+1}\right) \cdot \mathbf{x}_{j}=\left(\sum_{i=0}^{r-1} a_{i} \mathbf{x}_{i}\right) \cdot \mathbf{x}_{j-1}=\mathbf{x}_{N} \cdot \mathbf{x}_{j-1}=\mathbf{x}_{N+1-p} \cdot \mathbf{x}_{j-p},
$$

which proves the claim.

We will now consider two-dimensional examples. We will need the following result.

Lemma 5.9. Let $\Delta=\left\{(i, j) \in \mathbf{Z}^{2}, 0 \leq i \leq 1\right\}$ and, for any integer $r \geq 1$, let $\left\{\mathbf{x}_{\mathbf{k}}\right\}_{\mathbf{k} \in \Delta}$ be a collection of vectors in $\mathbf{C}^{r}$ satisfying $\mathbf{x}_{\mathbf{k}} \cdot \mathbf{x}_{\mathbf{l}}=\mathbf{x}_{\mathbf{p}} \cdot \mathbf{x}_{\mathbf{q}}$, for all $\mathbf{k}, \mathbf{l}, \mathbf{p}, \mathbf{q} \in$ $\Delta$ with $\mathbf{k}-\mathbf{l}=\mathbf{p}-\mathbf{q}$. Then, there exist two commuting unitary matrices $U_{1}, U_{2} \in \mathbf{M}_{r}$ such that $\mathbf{x}_{\mathbf{k}}=U_{1}^{k_{1}} U_{2}^{k_{2}} \mathbf{x}_{(0,0)}$, for all $\mathbf{k}=\left(k_{1}, k_{2}\right) \in \Delta$.

Proof. There is no loss of generality in assuming that the span of the collection $\left\{\mathbf{x}_{\mathbf{k}}\right\}_{\mathbf{k} \in \Delta}$ is all of $\mathbf{C}_{r}$. For any finite sum $\sum_{\mathbf{k} \in \Delta} a_{\mathbf{k}} \mathbf{x}_{\mathbf{k}}$, we define

$$
U_{2}\left(\sum_{\mathbf{k} \in \Delta} a_{\mathbf{k}} \mathbf{x}_{\mathbf{k}}\right)=\sum_{\mathbf{k} \in \Delta} a_{\mathbf{k}} \mathbf{x}_{\mathbf{k}+\mathbf{e}_{2}},
$$

where $\mathbf{e}_{2}=(0,1)$. It is clear that $U_{2}$ defines a unitary operator on $\mathbf{C}^{r}$ since

$$
\begin{aligned}
\left|U_{2}\left(\sum_{\mathbf{k} \in \Delta} a_{\mathbf{k}} \mathbf{x}_{\mathbf{k}}\right)\right|^{2}= & \left|\sum_{\mathbf{k} \in \Delta} a_{\mathbf{k}} \mathbf{x}_{\mathbf{k}+\mathbf{e}_{2}}\right|^{2}=\sum_{\mathbf{k}, \mathbf{l} \in \Delta} a_{\mathbf{k}} \overline{a_{\mathbf{l}}} \mathbf{x}_{\mathbf{k}+\mathbf{e}_{2}} \cdot \mathbf{x}_{\mathbf{l}+\mathbf{e}_{2}} \\
& =\sum_{\mathbf{k}, \mathbf{l} \in \Delta} a_{\mathbf{k}} \overline{a_{\mathbf{l}}} \mathbf{x}_{\mathbf{k}} \cdot \mathbf{x}_{\mathbf{l}}=\left|\sum_{\mathbf{k} \in \Delta} a_{\mathbf{k}} \mathbf{x}_{\mathbf{k}}\right|^{2} .
\end{aligned}
$$

Let $M_{i}$ be the span of $\left\{\mathbf{x}_{(i, j)}, j \in \mathbf{Z}\right\}$, for $i=0,1$. Let $V: M_{0} \rightarrow M_{1}$ be the mapping defined, for finite sums, by $V\left(\sum_{j \in \mathbf{Z}} a_{(0, j)} \mathbf{x}_{(0, j)}\right)=\sum_{j \in \mathbf{Z}} a_{(0, j)} \mathbf{x}_{(1, j)}$. One easily checks, as before, that $V$ is unitary. Let $\alpha_{1}, \ldots, \alpha_{s}, 1 \leq s \leq r$, be the distinct eigenvalues of $U_{2}$ and let $E_{1}, \ldots, E_{s}$ be the corresponding eigenspaces. Note that both $M_{0}$ and $M_{1}$ are invariant under $U_{2}$. The eigenvalues of the restriction of $U_{2}$ to $M_{0}$ are clearly contained in the set of eigenvalues of $U_{2}$. Let $\alpha_{j}$ be such an eigenvalue and let $\psi_{j}^{1}, \ldots, \psi_{j}^{k(j)}, 1 \leq k(j) \leq \operatorname{dim}\left(E_{j}\right)$, be an orthonormal basis for $E_{j} \cap M_{0}$, i.e. the eigenspace corresponding to $\alpha_{j}$ associated with the restriction of $U_{2}$ to $M_{0}$. Then, the collection $\left\{\phi_{j}^{i}, i=1, \ldots, k(j)\right\}$, where $\phi_{j}^{i}=V\left(\psi_{j}^{i}\right)$, is an orthonormal basis for $E_{j} \cap M_{1}$, since $V$ is unitary and the image under $V$ of an eigenvector of $U_{2}$ in $E_{j} \cap M_{0}$ is again an eigenvector of $U_{2}$ corresponding to the eigenvalue $\alpha_{j}$. If $E_{j} \cap M_{0}=\{0\}$ or if $k(j)<\operatorname{dim}\left(E_{j}\right)$, let us complete the orthonormal basis obtained for $E_{j} \cap M_{0}$ into an orthonormal basis $\left\{\psi_{j}^{i}, i=1, \ldots, \operatorname{dim}\left(E_{j}\right)\right\}$ for $E_{j}$, and similarly, let us complete the orthonormal basis obtained for $E_{j} \cap M_{1}$ into an orthonormal basis $\left\{\phi_{j}^{i}, i=1, \ldots, \operatorname{dim}\left(E_{j}\right)\right\}$ for $E_{j}$. Let $U_{1}: \mathbf{C}^{r} \rightarrow \mathbf{C}^{r}$ be the unitary operator defined by $U_{1}\left(\psi_{j}^{i}\right)=\phi_{j}^{i}$, for $j=1, \ldots, s$ and $i=1, \ldots, \operatorname{dim}\left(E_{j}\right)$. Then, clearly, $V$ is the restriction of $U_{1}$ to $M_{0}$, and $U_{1} U_{2}=U_{2} U_{1}$, since the restriction of $U_{1} U_{2}$ and $U_{2} U_{1}$ trivially agree on each $E_{j}, j=1, \ldots, s$. Since, as can be easily checked, $\mathbf{x}_{\mathbf{k}}=\mathbf{U}^{\mathbf{k}} \mathbf{x}_{(0,0)}$, for all $\mathbf{k} \in \Delta$, where $\mathbf{U}=\left(U_{1}, U_{2}\right)$, the result follows. 
A consequence of Theorem 4.9 is that the set $\Lambda=R(p, q)$ fails to satisfy the extension property if both $p, q \geq 2$. We will show next that the extension property holds if $\Lambda=R(1, p)$ (or $R(p, 1)$ ), for any $p \geq 1$. The case of $R(1,2)$ is already known (see $[\mathrm{Sa}]$ and the references therein).

Theorem 5.10. The set $\Lambda=R(1, p)$ satisfies the extension property, for any integer $p \geq 1$.

Proof. We have $|\Lambda|=2 p+2$. Let us consider a collection of unit vectors $\left\{\mathbf{x}_{\mathbf{k}}\right\}_{\mathbf{k} \in \Lambda}$ in $\mathbf{C}^{r}$ with $r \leq 2 p+1$, satisfying $\mathbf{x}_{\mathbf{k}} \cdot \mathbf{x}_{\mathbf{l}}=\mathbf{x}_{\mathbf{p}} \cdot \mathbf{x}_{\mathbf{q}}$, for all $\mathbf{k}, \mathbf{l}, \mathbf{p}, \mathbf{q} \in \Lambda$ with $\mathbf{k}-$ $\mathbf{l}=\mathbf{p}-\mathbf{q}$, and whose span is all of $\mathbf{C}^{r}$. Let $M$ be the span of the collection $\left\{\mathbf{x}_{(i, j)}, i=0,1 ; 0 \leq j \leq p-1\right\}$. We claim that the dimension of $M$ is at least $r-1$. Indeed, if it were less than or equal to $r-2 \leq 2 p-1$, the $2 p$ vectors in that collection would have to be linearly dependent. This would imply the existence of an integer $k$ with $0 \leq k \leq p-1$ such that either $\mathbf{x}_{(0, k)}$ belongs to the span of the collection $\left\{\mathbf{x}_{(i, j)}, i=0,1,0 \leq j<k\right\}$ or $\mathbf{x}_{(1, k)}$ belongs to the span of the collection $\left\{\mathbf{x}_{(0, k)}\right\} \cup\left\{\mathbf{x}_{(i, j)}, i=0,1,0 \leq j<k\right\}$. In the first case, we would have that

$$
\mathbf{x}_{(0, k)}=\sum_{\substack{i=0,1 \\ 0 \leq j<k}} a_{i j} \mathbf{x}_{(i, j)},
$$

for some complex numbers $a_{i j}$, and, thus, that

$$
\mathbf{x}_{(0, p)}=\sum_{\substack{i=0,1 \\ 0 \leq j<k}} a_{i j} \mathbf{x}_{(i, j+p-k)},
$$

since the mapping

$$
\sum_{\substack{i=0,1 \\ 0 \leq j \leq k}} b_{i j} \mathbf{x}_{(i, j)} \rightarrow \sum_{\substack{i=0,1 \\ 0 \leq j \leq k}} b_{i j} \mathbf{x}_{(i, j+p-k)}
$$

is norm preserving. This would contradict the fact that the collection $\left\{\mathbf{x}_{\mathbf{k}}\right\}_{\mathbf{k} \in \Lambda}$ spans all of $\mathbf{C}^{r}$. A similar argument shows that the second case is impossible as well. We define a unitary mapping $V$ from $M$ onto a subspace of $\mathbf{C}^{r}$ by

$$
V\left(\sum_{\substack{i=0,1 \\ 0 \leq j \leq p-1}} a_{i j} \mathbf{x}_{(i, j)}\right)=\sum_{\substack{i=0,1 \\ 0 \leq j \leq p-1}} a_{i j} \mathbf{x}_{(i, j+1)} .
$$

If $\operatorname{dim}(M)=r-1$, we choose unit vectors $\mathbf{y}, \mathbf{z}$ orthogonal to $M$ and $V(M)$, respectively, and define a unitary operator $U_{2}$ on $\mathbf{C}^{r}$ by $U_{2}(\mathbf{x}+\lambda \mathbf{y})=V(\mathbf{x})+\lambda \mathbf{z}$. If $\operatorname{dim}(M)=r$, i.e. $M=\mathbf{C}^{r}$, we simply define $U_{2}=V$. It is clear from this definition that $\mathbf{x}_{(0, j)}=U_{2}^{j}\left(\mathbf{x}_{(0,0)}\right)$ and $\mathbf{x}_{(1, j)}=U_{2}^{j}\left(\mathbf{x}_{(1,0)}\right)$, for all $j=0, \ldots, n$. Let us define $\mathbf{x}_{(i, j)}=U_{2}^{j}\left(\mathbf{x}_{(i, 0)}\right)$, for all $i=0,1$ and all $j \in \mathbf{Z}$. Let $\Delta=\{(i, j) \in$ $\left.\mathbf{Z}^{2}, 0 \leq i \leq 1, j \in \mathbf{Z}\right\}$. We claim that we have $\mathbf{x}_{\mathbf{k}} \cdot \mathbf{x}_{\mathbf{l}}=\mathbf{x}_{\mathbf{p}} \cdot \mathbf{x}_{\mathbf{q}}$, for all $\mathbf{k}, \mathbf{l}, \mathbf{p}, \mathbf{q} \in \Delta$ with $\mathbf{k}-\mathbf{l}=\mathbf{p}-\mathbf{q}$. Indeed, suppose that $\mathbf{k}=\left(i, j_{1}\right), \mathbf{l}=\left(i, j_{2}\right), \mathbf{p}=\left(i, j_{1}+m\right)$, $\mathbf{q}=\left(i, j_{2}+m\right)$, where $0 \leq i \leq 1$, then

$$
\mathbf{x}_{\mathbf{k}} \cdot \mathbf{x}_{\mathbf{l}}=U_{2}^{j_{1}} \mathbf{x}_{(i, 0)} \cdot U_{2}^{j_{2}} \mathbf{x}_{(i, 0)}=U_{2}^{j_{1}+m} \mathbf{x}_{(i, 0)} \cdot U_{2}^{j_{2}+m} \mathbf{x}_{(i, 0)}=\mathbf{x}_{\mathbf{p}} \cdot \mathbf{x}_{\mathbf{q}} \cdot
$$

Similarly, if $\mathbf{k}=\left(0, j_{1}\right), \mathbf{l}=\left(1, j_{2}\right), \mathbf{p}=\left(0, j_{1}+m\right), \mathbf{q}=\left(1, j_{2}+m\right)$, we have that

$$
\mathbf{x}_{\mathbf{k}} \cdot \mathbf{x}_{\mathbf{l}}=U_{2}^{j_{1}} \mathbf{x}_{(0,0)} \cdot U_{2}^{j_{2}} \mathbf{x}_{(1,0)}=U_{2}^{j_{1}+m} \mathbf{x}_{(0,0)} \cdot U_{2}^{j_{2}+m} \mathbf{x}_{(1,0)}=\mathbf{x}_{\mathbf{p}} \cdot \mathbf{x}_{\mathbf{q}} \cdot
$$


The only case which is nontrivial is when $\mathbf{k}=\left(0, j_{1}\right), \mathbf{l}=\left(0, j_{2}\right), \mathbf{p}=\left(1, j_{1}+m\right)$, $\mathbf{q}=\left(1, j_{2}+m\right)$, and this case can be immediately reduced, by the previous steps, to the case where $\mathbf{k}=(0,0), \mathbf{l}=(0, j), \mathbf{p}=(1,0), \mathbf{q}=(1, j)$. We need thus to show that

$$
U_{2}^{j} x_{(0,0)} \cdot x_{(0,0)}=U_{2}^{j} x_{(1,0)} \cdot x_{(1,0)},
$$

for all integers $j$, knowing that this identity holds when $0 \leq j \leq p$ and, thus, when $|j| \leq p$. Let $\phi_{1}, \ldots, \phi_{r}$ be an orthonormal basis of eigenvectors of $U_{2}$ and let $\alpha_{1}, \ldots, \alpha_{r} \in \mathbf{T}$ be the corresponding eigenvalues. If $x_{(0,0)}=\sum_{1 \leq i \leq r} a_{i} \phi_{i}$ and $x_{(1,0)}=\sum_{1 \leq i \leq r} b_{i} \phi_{i}$, then

$$
U_{2}^{j} x_{(0,0)} \cdot x_{(0,0)}=\sum_{1 \leq i \leq r} \alpha_{i}^{j}\left|a_{i}\right|^{2}
$$

and

$$
U_{2}^{j} x_{(1,0)} \cdot x_{(1,0)}=\sum_{1 \leq i \leq r} \alpha_{i}^{j}\left|b_{i}\right|^{2} .
$$

Consider the positive measures $\mu=\sum_{1 \leq i \leq r}\left|a_{i}\right|^{2} \delta_{\alpha_{i}}$ and $\nu=\sum_{1 \leq i \leq r}\left|b_{i}\right|^{2} \delta_{\alpha_{i}}$ defined on $\mathbf{T}$. It follows from our assumptions that if $\Lambda_{1}=\{j \in \mathbf{Z},|j| \leq p\}$, then

$$
\int_{\mathbf{T}} P d \mu=\int_{\mathbf{T}} P d \nu
$$

for all $P \in \Pi_{\Lambda_{1}}$. Since $r \leq 2 p+1$, we can find, for each $i=1, \ldots, r$, using the Lagrange interpolation formula, a polynomial $P_{i} \in \Pi_{\Lambda_{1}}$ such that $P_{i}\left(\alpha_{i}\right)=1$ and $P_{i}\left(\alpha_{j}\right)=0$, for all $j \in\{0, \ldots, r\}$ with $j \neq i$. Replacing $P$ by $P_{i}$ in (5.10), we deduce that $\left|a_{i}\right|=\left|b_{i}\right|$ for all $i=1, \ldots, r$. Hence, $\mu=\nu$ and we have thus equality between the expressions in (5.8) and (5.9) for all $j \in \mathbf{Z}$, which proves the claim. To conclude the proof, it suffices now to apply Lemma 5.9 to the collection $\left\{\mathbf{x}_{\mathbf{k}}\right\}_{\mathbf{k} \in \Delta}$ we have constructed.

\section{EXPLICIT EXAMPLES OF NON-EXTENDABLE COLLECTIONS}

We will use the methods developed so far in this paper to construct explicit examples of collections that are p.d., but are not extendable, on the sets $\Lambda=R(p, q)$, where $p, q \geq 2$ and $\Lambda=R(p, q, r)$, where $p, q, r \geq 1$. Explicit examples, although not as simple as the ones given below, can be found in [Sa] for the case $\Lambda=R(2,2)$ and $\Lambda=R(1,1,1)$, and an algorithm describing how to construct such collections from the knowledge of a non-negative polynomial in $\Pi_{\Lambda-\Lambda}$ that cannot be written as a sum of squares of moduli of polynomials in $\Pi_{\Lambda}$ is given in [Lan].

Theorem 6.1. If $p, q \geq 2$ and $\Lambda=R(p, q)$, the collection $\left\{c_{\mathbf{k}}\right\}_{\mathbf{k} \in \Lambda-\Lambda}$ defined by

$$
c_{\mathbf{k}}=r_{\mathbf{k}}-(p q+p+q-1)^{-1}, \quad \mathbf{k} \in \Lambda-\Lambda,
$$

where $r_{(0,0)}=r_{( \pm p, \pm q)}=1$ and $r_{(i, j)}=0$ otherwise, is $p . d$. on $\Lambda$, but not extendable.

Proof. Let $\Lambda_{1}=\Lambda \backslash\{(0,0),(p, 0),(0, q),(p, q)\}$. If $P\left(z_{1}, z_{2}\right)=\sum_{(i, j) \in \Lambda} a_{(i, j)} z_{1}^{i} z_{2}^{j} \in$ $\Pi_{\Lambda}$, we define

$$
\|P\|^{2}=\sum_{(i, j) \in \Lambda_{1}}\left|a_{(i, j)}\right|^{2}+\left|a_{(0,0)}+a_{(p, q)}\right|^{2}+\left|a_{(p, 0)}+a_{(0, q)}\right|^{2}=\sum_{\mathbf{k}, \mathbf{l} \in \Lambda} r_{\mathbf{l}-\mathbf{k}} a_{\mathbf{k}} \overline{a_{\mathbf{l}}},
$$




$$
M=\left\{P \in \Pi_{\Lambda},\|P\|=0\right\}=\left\{c_{1}\left(1-z_{1}^{p} z_{2}^{q}\right)+c_{2}\left(z_{1}^{p}-z_{2}^{q}\right), c_{1}, c_{2} \in \mathbf{C}\right\},
$$

and

$$
\Omega=\left\{\alpha \in \mathbf{T}^{2}, P(\alpha)=0, \forall P \in M\right\}=\left\{\left(\alpha_{1}, \alpha_{2}\right) \in \mathbf{T}^{2}, \alpha_{1}^{p}=\alpha_{2}^{q}, \alpha_{2}^{2 q}=1\right\} .
$$

If $\alpha \in \Omega$, we consider the evaluation polynomials $E_{\alpha}$ which are defined up to an element of $M$. If $P \in \Pi_{\Lambda}$, we have

$$
P(1,1)=\sum_{(i, j) \in \Lambda} a_{(i, j)}=\sum_{(i, j) \in \Lambda_{1}} a_{(i, j)}+\left(a_{(0,0)}+a_{(p, q)}\right)+\left(a_{(p, 0)}+a_{(0, q)}\right) .
$$

Hence we can define

$$
\begin{aligned}
E_{(1,1)}\left(z_{1}, z_{2}\right) & =\sum_{(i, j) \in \Lambda_{1}} z_{1}^{i} z_{2}^{j}+\frac{1}{2}\left(1+z_{1}^{p} z_{2}^{q}\right)+\frac{1}{2}\left(z_{1}^{p}+z_{2}^{q}\right) \\
& =\sum_{(i, j) \in \Lambda} z_{1}^{i} z_{2}^{j}-\frac{1}{2}\left(1+z_{1}^{p} z_{2}^{q}\right)-\frac{1}{2}\left(z_{1}^{p}+z_{2}^{q}\right) \\
& =\left(\sum_{i=0}^{p} z_{1}^{i}\right)\left(\sum_{j=0}^{q} z_{2}^{j}\right)-\frac{1}{2}\left(1+z_{1}^{p} z_{2}^{q}\right)-\frac{1}{2}\left(z_{1}^{p}+z_{2}^{q}\right) .
\end{aligned}
$$

Suppose now that $\beta=\left(\beta_{1}, \beta_{2}\right)$ belongs to $\Omega$. If $\beta_{1}^{p}=\beta_{2}^{q}=-1$, then

$$
E_{(1,1)}\left(\beta_{1}, \beta_{2}\right)=\left(\frac{1+\beta_{1}}{1-\beta_{1}}\right)\left(\frac{1+\beta_{2}}{1-\beta_{2}}\right) .
$$

Thus, $E_{(1,1)}(\beta)=0$ only in the case where $\beta_{1}=-1$ or $\beta_{2}=-1$. The first case can only occur when $p$ is odd and yields $q$ zeros in $\Omega$ of the form $\left(-1, \beta_{2}\right)$, where $\beta_{2}^{q}=-1$. The second case occurs only when $q$ is odd and yields $p$ zeros of the form $\left(\beta_{1},-1\right)$, where $\beta_{1}^{p}=-1$. If $\beta_{1}^{p}=\beta_{2}^{q}=1$, we obtain that $E_{(1,1)}(\beta)=-1$ when $\beta_{1} \neq 1$ and $\beta_{2} \neq 1, E_{(1,1)}(\beta)=p-1 \neq 0$ when $\beta_{1}=1$ and $\beta_{2} \neq 1$, and $E_{(1,1)}(\beta)=q-1 \neq 0$ when $\beta_{1} \neq 1$ and $\beta_{2}=1$. So $E_{(1,1)}$ has at most $p+q$ zeros in $\Omega$. If $P(\mathbf{z})=\sum_{\mathbf{k} \in \Lambda} a_{\mathbf{k}} \mathbf{z}^{\mathbf{k}} \in \Pi_{\Lambda}$, we have that

$$
\sum_{\mathbf{k}, \mathbf{l} \in \Lambda} c_{\mathbf{l}-\mathbf{k}} a_{\mathbf{k}} \overline{a_{\mathbf{l}}}=\|P\|^{2}-\frac{|P(1,1)|^{2}}{\left\|E_{(1,1)}\right\|^{2}} \geq 0
$$

and, thus, the collection $\left\{c_{\mathbf{k}}\right\}_{\mathbf{k} \in \Lambda-\Lambda}$ is p.d. on $\Lambda$. If that collection were extendable, any associated representing measure $\mu$ would have to be supported in the intersection of $\Omega$ with the zero set of $E_{(1,1)}$ (see the proof of Theorem 3.9) and would therefore be a measure supported on a set of cardinality at most $p+q$. The dimension of the subset of $\Pi_{\Lambda}$ consisting of the polynomials in $\Pi_{\Lambda}$ vanishing on the support of $\mu$ has to be at least $|\Lambda|-(p+q)$ while, on the other hand, the dimension of the set of polynomials in $\Pi_{\Lambda}$ for which the left-hand side of (6.1) vanishes is 3 . Hence, $(p+1)(q+1)-(p+q) \leq 3$ or $p q \leq 2$, which contradicts the fact that both $p$ and $q$ are greater than or equal to 2 . Therefore, $\left\{c_{\mathbf{k}}\right\}_{\mathbf{k} \in \Lambda-\Lambda}$ is not extendable.

Remark 6.2. The collection $\left\{r_{\mathbf{k}}\right\}_{\mathbf{k} \in \Lambda-\Lambda}$ defined in the previous theorem is itself extendable. Indeed, it is easily checked that a representing measure associated with that collection is the measure

$$
\mu=\frac{(2 \pi)^{2}}{2(p+1)(q+1)} \sum_{k=0}^{p} \sum_{l=0}^{q} \delta_{\left(\beta_{1}^{k}, \beta_{2}^{l}\right)}+\delta_{\left(\gamma_{1} \beta_{1}^{k}, \gamma_{2} \beta_{2}^{l}\right)},
$$

where $\beta_{1}=e^{2 \pi i / p}, \gamma_{1}=e^{\pi i / p}, \beta_{2}=e^{2 \pi i / q}$, and $\gamma_{2}=e^{\pi i / q}$. 
Theorem 6.3. If $p, q, r \geq 1$ and $\Lambda=R(p, q, r)$, the collection $\left\{c_{\mathbf{k}}\right\}_{\mathbf{k} \in \Lambda-\Lambda}$ defined by

$$
c_{\mathbf{k}}=r_{\mathbf{k}}-[(p+1)(q+1)(r+1)-3]^{-1}, \quad \mathbf{k} \in \Lambda-\Lambda,
$$

where $r_{(0,0,0)}=r_{(p,-q,-r)}=r_{(-p, q, r)}=r_{(-p, q,-r)}=r_{(p,-q, r)}=r_{(-p,-q, r)}=$ $r_{(p, q,-r)}=1$ and $r_{(i, j, k)}=0$ otherwise, is p.d. on $\Lambda$, but not extendable.

Proof. Let $\Lambda_{1}=\Lambda \backslash\{(p, 0,0),(0, q, 0),(0,0, r),(p, q, 0),(p, 0, r),(0, q, r)\}$. For the polynomial $P\left(z_{1}, z_{2}, z_{3}\right)=\sum_{(i, j, k) \in \Lambda} a_{(i, j, k)} z_{1}^{i} z_{2}^{j} z_{3}^{k} \in \Pi_{\Lambda}$, we define

$$
\begin{aligned}
\|P\|^{2}= & \sum_{(i, j, k) \in \Lambda_{1}}\left|a_{(i, j, k)}\right|^{2}+\left|a_{(p, 0,0)}+a_{(0, q, r)}\right|^{2}+\left|a_{(0, q, 0)}+a_{(p, 0, r)}\right|^{2} \\
& \quad+\left|a_{(0,0, r)}+a_{(p, q, 0)}\right|^{2} \\
= & \sum_{\mathbf{k}, \mathbf{l} \in \Lambda} r_{\mathbf{l}-\mathbf{k}} a_{\mathbf{k}} \overline{a_{\mathbf{l}}} .
\end{aligned}
$$

We also define the sets

$$
\begin{aligned}
M & =\left\{P \in \Pi_{\Lambda},\|P\|=0\right\} \\
& =\left\{c_{1}\left(z_{1}^{p}-z_{2}^{q} z_{3}^{r}\right)+c_{2}\left(z_{2}^{q}-z_{1}^{p} z_{3}^{r}\right)+c_{3}\left(z_{3}^{r}-z_{1}^{p} z_{2}^{q}\right), c_{1}, c_{2}, c_{3} \in \mathbf{C}\right\},
\end{aligned}
$$

and

$$
\Omega=\left(\alpha \in \mathbf{T}^{3}, P(\alpha)=0, \forall P \in M\right\}=\left\{\left(\alpha_{1}, \alpha_{2}, \alpha_{3}\right) \in \mathbf{T}^{3},\left(\alpha_{1}^{p}, \alpha_{2}^{q}, \alpha_{3}^{r}\right) \in F\right\},
$$

where $F=\{(1,1,1),(1,-1,-1),(-1,1,-1),(-1,-1,1)\}$. Similarly to the previous theorem, it is easily checked that the evaluation polynomial at the point $(1,1,1) \in \Omega$ can be taken to be

$$
\begin{aligned}
& E_{(1,1,1)}\left(z_{1}, z_{2}, z_{3}\right) \\
& =\left(\sum_{i=0}^{p} z_{1}^{i}\right)\left(\sum_{j=0}^{q} z_{2}^{j}\right)\left(\sum_{k=0}^{r} z_{3}^{k}\right)-\frac{1}{2}\left\{z_{1}^{p}+z_{2}^{q}+z_{3}^{r}+z_{1}^{p} z_{2}^{q}+z_{1}^{p} z_{3}^{r}+z_{2}^{q} z_{3}^{r}\right\} .
\end{aligned}
$$

Notice also that, if $P(\mathbf{z})=\sum_{\mathbf{k} \in \Lambda} a_{\mathbf{k}} \mathbf{z}^{\mathbf{k}} \in \Pi_{\Lambda}$, we have that

$$
\sum_{\mathbf{k}, \mathbf{l} \in \Lambda} c_{\mathbf{l}-\mathbf{k}} a_{\mathbf{k}} \overline{a_{\mathbf{l}}}=\|P\|^{2}-\frac{|P(1,1,1)|^{2}}{\left\|E_{(1,1,1)}\right\|^{2}} \geq 0,
$$

so that the collection $\left\{c_{\mathbf{k}}\right\}_{\mathbf{k} \in \Lambda}$ is p.d. on $\Lambda$. Furthermore, any representing measure associated with the collection $\left\{c_{\mathbf{k}}\right\}_{\mathbf{k} \in \Lambda}$ would have to be supported in the intersection of $\Omega$ with the zero-set of $E_{(1,1,1)}$ (see the proof of Theorem 6.1). Also, since $\operatorname{dim} M=4$, any representing measure would have to have at least $|\Lambda|-4$ points in its support. To prove the theorem, it is thus enough to verify that the number of zeros of $E_{(1,1,1)}$ in $\Omega$ is less than $|\Lambda|-4=(p+1)(q+1)(r+1)-4$. Suppose thus that $\beta=\left(\beta_{1}, \beta_{2}, \beta_{3}\right)$ belongs to $\Omega$. If $\beta_{1}^{p}=\beta_{2}^{q}=\beta_{3}^{r}=1$, it is easily checked that $E_{(1,1,1)}\left(\beta_{1}, \beta_{2}, \beta_{3}\right) \neq 0$, unless $p=2, q=2$, or $r=2$. The first case yields $(q-1)(r-1)$ zeros of the form $\left(1, \beta_{2}, \beta_{3}\right)$, where $\beta_{2}^{q}=\beta_{3}^{r}=1$, $\beta_{2} \neq 1$, and $\beta_{1} \neq 1$. Similarly, the second case yields $(p-1)(r-1)$ zeros while the third one yields $(p-1)(q-1)$ zeros. If $\beta_{1}^{p}=1$ and $\beta_{2}^{q}=\beta_{3}^{r}=-1$, the equation $E_{(1,1,1)}\left(\beta_{1}, \beta_{2}, \beta_{3}\right)=0$ reduces to

$$
\beta_{3}=-\frac{(p+2)+p \beta_{2}}{p+(p+2) \beta_{2}}
$$


when $\beta_{1}=1$, and to

$$
\beta_{3}=-\overline{\beta_{2}},
$$

when $\beta_{1} \neq 1$. The equations for the cases $\beta_{2}^{q}=1, \beta_{1}^{p}=\beta_{3}^{r}=-1$, and $\beta_{3}^{r}=1, \beta_{1}^{p}=$ $\beta_{2}^{q}=-1$ are similar. If $p, q$ and $r$ are all different from 2 , a very rough estimate shows that the number of zeros of $E_{(1,1,1)}$ in $\Omega$ is not greater than

$$
p \min (q, r)+q \min (p, r)+r \min (p, q) \leq p q+p r+q r
$$

while

$$
(p+1)(q+1)(r+1)-4=p q r+p+q+r-3+p q+p r+q r>p q+p r+q r .
$$

We now treat the case where exactly one of $p, q$ or $r$ is equal to 2 . Let us say, for example, that $p=2$. In that case, the number of zeros of $E_{(1,1,1)}$ in $\Omega$ is not greater than

$$
(q-1)(r-1)+2 \min (q, r)+q \min (2, r)+r \min (2, q) \leq 3 q r+1
$$

while

$$
|\Lambda|-4=3(q+1)(r+1)-4=3 q r+3(q+r)-1>3 q r+1 .
$$

If $p=q=2$, and $r \neq 2$, the same rough estimate as above shows that the number of zeros of $E_{(1,1,1)}$ in $\Omega$ is not greater than $4 r-2+4 \min (2, r) \leq 4 r+6$, while $|\Lambda|-4=9 r+5$ proving the desired inequality in that case. The other cases where exactly two of $p, q$ and $r$ are equal to 2 are handled in a similar way. Finally, if $p=q=r=2$, one can check directly that (6.2) and its analogues in the other variables have no solution while (6.3) and its analogues each have two solutions. The exact number of zeros of $E_{(1,1,1)}$ in $\Omega$ is thus 9 while $|\Lambda|-4=23>9$, which concludes the proof.

Remark 6.4. All examples constructed in this section are of collections $\left\{c_{\mathbf{k}}\right\}_{\mathbf{k} \in \Lambda-\Lambda}$ which are p.d., but not s.p.d., on $\Lambda$. To obtain examples of s.p.d. collections $\left\{b_{\mathbf{k}}\right\}_{\mathbf{k} \in \Lambda-\Lambda}$ which are not extendable, it suffices to define $b_{0}=c_{0}+\epsilon(\Lambda)$ and $b_{\mathbf{k}}=c_{\mathbf{k}}$ otherwise, where $\epsilon(\Lambda)>0$ is small enough, as a weak-star compactness argument easily shows.

\section{ACKNOWLEDGMENT}

The author would like to thank the referee for valuable comments that helped improve the presentation of this paper as well as the Centre de Recherche Mathématique of the University of Montréal and the University of Mons, Hainaut, for their hospitality while this manuscript was being prepared.

\section{REFERENCES}

[Ak] N. I. Akhiezer, The Classical Moment Problem, Oliver and Boyd, Edinburgh, 1965. MR 32:1518

[AK] N. I. Akhiezer and M. Krein, Somes Questions in the Theory of Moments, Transl. Math. Monographs, vol.2, Amer. Math. Soc., Providence, 1962. MR 29:5073

[Be] J. J. Benedetto, Irregular sampling and frames, in Wavelets, a Tutorial in Theory and Applications (C. K. Chui, ed.), Academic Press, Boston, 1992, pp. 445-507. MR 93c:42030

$[\mathrm{CF}] \quad$ R. E. Curto and L. A. Fialkow, Recursiveness, positivity and truncated moment problems, Houston J. of Math. 17 (1991), 603-635. MR 93a:47016 
[CP] A. Calderón and R. Pepinsky, On the phases of Fourier coefficients for positive real periodic functions, in Computing Methods and the Phase Problem in X-Ray Crystal Analysis, The X-Ray Crystal Analysis Laboratory, Department of Physics, The Pennsylvannia State College, 1952, pp. 339-348.

[Da] I. Daubechies, Ten Lectures on Wavelets, CBMS 61, SIAM, Philadelphia, 1992. MR 93e: 42045

[DGM] I. Daubechies, A. Grossman, Y. Meyer, Painless nonorthogonal expansions, J. Math. Phys. 27 (1986), 1271-1283. MR 87e:81089

[De] A. Devinatz, On the extensions of positive definite functions, Acta Math. 102 (1959), 109-134. MR 22:875

[DS] R. Duffin and A. Schaeffer, A class of nonharmonic Fourier series, Trans. Amer. Math. Soc. 72 (1952), 341-366. MR 13:839a

[Fu] B. Fuglede, Commuting self-adjoint partial differential operators and a group theoretic problem, J. Funct. Anal. 16 (1974), 101-121. MR 57:10500

[Ga1] J.-P. Gabardo, Tight frames of polynomials and the truncated trigonometric moment problem, J. Fourier Anal. and Appl. 1 (1995), 249-279. MR 96m:42013

[Ga2] Extension of Positive-Definite Distributions and Maximum Entropy, Memoirs of the Amer. Math. Soc., no. 489, Amer. Math. Soc., Providence, 1993. MR 93g:42005

[HJ] R. A. Horn and C. R. Johnson, Matrix Analysis, Cambridge University Press, 1985. MR 87e: 15001

[Jo1] P. E. T. Jorgensen, Integral representations for locally defined positive definite functions on Lie groups, Inter. J. of Math. 2 (1991), 257-286. MR 92h:43017

[Jo2] _ Extensions of positive definite integral kernels on the Heisenberg group, J. Funct. Anal. 92 (1990), 474-508. MR 91 m:22013

[JP] P. E. T. Jorgensen and S. Pedersen, Spectral Theory for Borel Sets in $\mathbf{R}^{n}$ of finite measure, J. Funct. Anal. 107 (1992), 72-104. MR 93k:47005

[Kr] M. G. Krein, Sur le problème du prolongement des fonctions hermitiennes positives et continues, C. R. (Doklady) Acad. Sci. URSS (N. S.) 26 (1940), 17-22. MR 2:361h

[La1] H. Landau, The classical moment problem: Hilbertian methods, J. Functional Anal. 38 (1980), 255-272. MR 81i:44008

[La2] Maximum entropy and the moment problem, Bull. Amer. Math. Soc. 16 (1987), 47-77. MR 88k:42010

[Lan] S. W. Lang, A positive-definite matrix which is not extendable, IEEE Trans. Acoust., Speech, Signal Processing, ASSP-32 (1984), 930-932.

[Ru] W. Rudin, The extension problem for positive-definite functions, Illinois J. Math. 7 (1963), 532-539. MR 27:1779

[Sa] L. A. Sakhnovich, Effective construction of nonextendable Hermitian-positive functions of several variables, Funkts. Anal. Prilozh. 14 1980, 55-60 (Russian); English transl. in Funct. Anal. Appl. 14 1980, 290-294. MR 82j:49036

[Sas1] Z. Sasvári, Decomposition of positive definite functions defined on a neighborhood of the identity, Monat. Math. 104 (1987), 139-148. MR 89a:43007

[Sas2] _ On the extension of positive definite functions, Radovi Mat. 3 (1987), 235-240. MR 89c: 43010

[Sas3] _ Positive definite and definitizable functions, Akademie Verlag, Berlin, 1994. MR 95c: 43005

Department of Mathematics and Statistics, McMaster University, Hamilton, OnTARIO, L8S 4K1, CANADA

E-mail address: gabardo@mcmail.cis.mcmaster.ca 